\title{
Transverse momentum broadening of vector boson production in high energy nuclear collisions
}

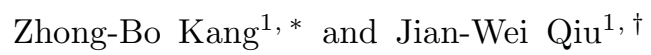 \\ ${ }^{1}$ Department of Physics and Astronomy, Iowa State University, Ames, IA 50011, U.S.A.
}

(Dated: August 11, 2021)

\begin{abstract}
We calculate in perturbative QCD the transverse momentum broadening of vector boson production in high energy nuclear collisions. We evaluate the effect of initial-state parton multiple scattering for the production of the Drell-Yan virtual photon and $W / Z$ bosons. We calculate both the initial- and final-state multiple scattering effect for the production of heavy quarkonia and their transverse momentum broadening in both NRQCD and Color Evaporation model of quarkonium formation. We find that $\mathrm{J} / \psi$ and $\Upsilon$ broadening in hadron-nucleus collision is close to $2 C_{A} / C_{F}$ times the corresponding Drell-Yan broadening, which gives a good description of existing Fermilab data. Our calculations are also consistent with RHIC data on $\mathrm{J} / \psi$ broadening in relativistic heavy ion collisions. We predict the transverse momentum broadening of vector boson $(\mathrm{J} / \psi, \Upsilon$, and $W / Z)$ production in relativistic heavy ion collisions at the LHC, and discuss the role of the vector boson broadening in diagnosing medium properties.
\end{abstract}

PACS numbers: 12.38.Bx, 12.39.St, 14.40.Gx, 24.85.+p

\section{INTRODUCTION}

The Relativistic Heavy Ion Collider (RHIC) at Brookhaven National Laboratory has been providing experimenters with colliding beams of heavy nuclei at relativistic energies as high as $100 \mathrm{GeV}$ per nucleon. Early RHIC data from central gold-gold collisions strongly indicate the creation of dense and hot QCD matter with very unusual and interesting properties [1]. Characterized by its opacity to jets and other evidences, the new QCD matter shows an unprecedented energy density well above the critical value predicted by lattice QCD for establishing quark-gluon plasma (QGP), a weakly coupled gas of quarks and gluons [2]. On the other hand, the data from the observation of an unexpectedly large flow indicate that the hot QCD matter interacts strongly with itself and behaves like an almost ideal liquid with low shear viscosity rather than a gas 11. In this paper, we investigate the role of transverse momentum broadening of heavy vector boson production in identifying the formation of new QCD matter and in diagnosing medium properties.

Hadronic production of a heavy vector boson, such as a virtual photon with a large invariant mass, a heavy quarkonium, and a $Z$ (or a $W$ ) boson requires a short-distance hard partonic collision with a large momentum transfer because of the large vector boson mass, and is dominated by the scattering of two partons from two incoming hadron beams (or from a beam and a target). The short-distance creation of the vector boson or the heavy quark pair could be perturbatively calculated in Quantum Chromodynamics (QCD), while the hadronization of the quark pair to a physical quarkonium offers unique perspective into the formation of QCD bound states. Heavy quarkonium production in high energy nuclear collisions is of special interest in diagnosing the properties of nuclear medium because of the two-scale nature of the creation process. The creation of the heavy quark pair takes place at such a short time that it is unlikely to interfere with the dynamics of nuclear medium which is effectively frozen. On the other hand, the hadronization from the heavy quark pair to a bound quarkonium could be very sensitive to the medium properties. A change in production rate and momentum spectrum for heavy quarkonium production from a proton-proton collision to a nuclear collision signals a change of characteristics of the nuclear matter and provides the opportunities to probe the matter's properties [3, 4]. The challenge is to quantitatively extract interesting properties of the nuclear matter from the change.

In high energy hadron-nucleus and nucleus-nucleus collisions, the interaction between the produced heavy quark pair and the nuclear medium (referred to as a final-state effect), as well as possible scattering between the incoming colliding partons and the nuclear matter before the hard collision to produce the pair (an initial-state effect), can both change the heavy quarkonium's production rate and its momentum spectrum. The effect of the final-state interaction depends on the hadronization mechanism - how a produced heavy quark pair becomes a bound quarkonium. A color

\footnotetext{
*Electronic address: kangzb@iastate.edu
}

${ }^{\dagger}$ Electronic address: jwq@iastate.edu 
singlet heavy quark pair with a small color dipole moment is less likely to interact with the nuclear medium than a pair in a color octet configuration [5, 6]. That is, the final-state effect could be an ideal probe for exploring the nonperturbative formation mechanism in heavy quarkonium production. But, the observed nuclear dependence is sensitive to not only the final-state effect but also the initial-state effect as well as the quantum interference between them. Due to the local hard collision that produces the heavy quark pair, the quantum interference between the finalstate and initial-state partonic rescattering could be suppressed by the hard collision scale. To understand the effect of the initial-state interaction, we also study nuclear dependence in the production of the Drell-Yan virtual photon as well as $Z$ (or $W$ ) vector boson. These vector bosons, if reconstructed from their leptonic decays, do not interact strongly once produced at the short-distance. The initial-state effect itself can be a good probe for the dynamics of partonic rescattering when a fast parton passes through the nuclear matter.

Partonic multiple rescattering in a nuclear medium before as well as after the hard collision can modify the distribution of the vector boson's transverse momentum $q_{T}$. Each rescattering is likely to change the momentum spectrum by an order of the typical transverse momentum of the partons inside the nuclear matter, which is significantly softer than the momentum exchange in the hard collision. Therefore, the change to the transverse momentum spectrum, $d \sigma / d q_{T}^{2}$, should be most significant when $q_{T}$ is relatively small. But, the rescattering effect on the low $q_{T}$ spectrum is unlikely to be calculable in perturbative QCD [7, 8, 9, 10]. On the other hand, an averaged transverse momentum square of the produced heavy vector boson,

$$
\left\langle q_{T}^{2}\right\rangle \equiv \int d q_{T}^{2} q_{T}^{2} \frac{d \sigma_{h h \rightarrow V^{*}}}{d q_{T}^{2}} / \int d q_{T}^{2} \frac{d \sigma_{h h \rightarrow V^{*}}}{d q_{T}^{2}},
$$

is much more inclusive and perturbatively calculable if the $q_{T}$ is integrated over a wide range [7, 9, 10, 11]. The accumulative change from the rescattering to the averaged transverse momentum square - transverse momentum broadening, $\left.\Delta\left\langle q_{T}^{2}\right\rangle \equiv\left\langle q_{T}^{2}\right\rangle\right|_{A B}-\left.\left\langle q_{T}^{2}\right\rangle\right|_{h h}$, defined as a difference between the calculable transverse momentum square in nuclear collision and that in hadron-hadron collision, should be calculable too [7, 9].

The Drell-Yan transverse momentum broadening $\Delta\left\langle q_{T}^{2}\right\rangle_{\text {DY }}$ has been studied in perturbative QCD by evaluating coherent partonic rescattering diagrams between incoming (anti)quark and the cold nuclear matter [12, 13, 14]. The rescattering leads to the broadening of the Drell-Yan dilepton's transverse momentum distribution. The broadening was shown to be proportional to the target size or to have the $A^{1 / 3}$-type nuclear dependence. The calculated nuclear dependence was found to be consistent with both Fermilab and CERN data [13]. On the other hand, as shown in Ref. [15], there have been difficulties in understanding the same broadening in the production of heavy quarkonia $(\mathrm{J} / \psi$ and $\Upsilon)$. At the leading order of perturbative calculation, the Drell-Yan dilepton production is dominated by a quark-antiquark annihilation subprocess, while the heavy quarkonium production in hadronic collision is dominated by a gluon-gluon fusion subprocess. If one neglected final-state interaction in both Drell-Yan and heavy quarkonium production, one would expect that the ratio of broadening of heavy quarkonium over Drell-Yan is close to the ratio of the multiple scattering effect of a gluon over that of a quark (or an antiquark) inside a nuclear medium [16]. At the lowest order, the ratio is approximately equal to the ratio of color factors of the lowest order gluon and (anti)quark rescattering, $C_{A} / C_{F}=9 / 4$, with $C_{A}=N_{c}=3$ and $C_{F}=\left(N_{c}^{2}-1\right) /\left(2 N_{c}\right)=4 / 3$. Although the data on heavy quarkonium broadening in hadron-nucleus collisions shows the expected $A^{1 / 3}$-type nuclear dependence, the ratio to the Drell-Yan broadening could be as large as 5, twice of the naive expectation [15].

Recently, Johnson et al. published in Ref. [17] a new analysis of Drell-Yan broadening, $\Delta\left\langle q_{T}^{2}\right\rangle_{D Y}$, based on the ratio of Drell-Yan transverse momentum distribution on heavy nuclear targets over deuterium target, $R_{A / D}$. By using the chain relation, $R_{A / D}=R_{A / B e} R_{B e / D}$, the new data from Fermilab E866 experiment on the ratio $R_{A / B e}$ with $A=F e, W$ over beryllium $(B e)$, and a theoretical formula for $R_{B e / D}$, Johnson et al. showed the result of their analysis in Fig. 5 of Ref. [17] and concluded that the observed broadening is about twice as large as the one published previously [15], which implies that the heavy quarkonium broadening is just a factor of $C_{A} / C_{F}$ larger than the Drell-Yan broadening and is consistent with the naive expectation. The factor of two enhancement of the Drell-Yan broadening represents a difference of more than three standard deviations between the central values of two different analyses in terms of the error size of previous analysis or a difference of two standard deviations if one uses the much larger error of the new analysis. It will be interesting to see if this new analysis changes the broadening of $\mathrm{J} / \psi$ and $\Upsilon$ as well. If the ratio of the heavy quarkonium broadening over Drell-Yan broadening is indeed consistent with the naive expectation, we would have to conclude based on the discussion on final-state interaction in this paper that the heavy quark pair in heavy quarkonium production is mainly produced in a color singlet state, and the color of an octet pair will have to be neutralized at a very short distance. This conclusion would have a very important impact on the physics of $\mathrm{J} / \psi$ suppression in nuclear medium.

Since a heavy quarkonium is unlikely to be formed at the same time when the heavy quark pair was produced [18], the final-state interaction between the heavy quark pair and the nuclear medium could generate additional broadening. Since the broadening is evaluated from the transverse momentum distribution of observed heavy quarkonia and normalized by the total production rate, as defined in Eq. (1), we will not discuss the impact of final-state interaction 
on the suppression of heavy quarkonium production in this paper [4, 5]. Since the final-state interaction is sensitive to the non-perturbative formation mechanism of the bound state, we calculate the broadening of heavy quarkonium production in both non-relativistic QCD (NRQCD) model [19] and Color Evaporation model 20]. The often used Color Singlet model [21] can be interpreted as a special case of the NRQCD model. In the NRQCD model, the nonperturbative dynamics for a heavy quark pair to form a bound quarkonium is organized through matrix elements of operators that are characterized by an expansion in the relative velocity of the pair and the pair's rotational and color quantum numbers. On the other hand, in the Color Evaporation model, all heavy quark pairs with invariant mass less than the mass threshold of producing a pair of open flavor heavy mesons have the same probability to become a bound quarkonium regardless the pair's rotational and color quantum numbers. Rescattering of the heavy quark pair in nuclear medium could change the pair's rotational and color quantum numbers. Therefore, these two models could lead to different predictions for the nuclear dependence of heavy quarkonium production in nuclear collisions. If the difference is significant, an accurate measurement of the nuclear dependence could provide important information on the hadronization. We find that the heavy quarkonium broadening calculated in these two models have different analytical expressions. But, numerically, these two models predict a very similar result for the broadening of $\mathrm{J} / \psi$ and $\Upsilon$ in hadron-nucleus collision. The calculated broadening is close to $2 C_{A} / C_{F}$, which is consistent with Fermilab data $[15,22]$.

We also investigate the nuclear dependence of the averaged transverse momentum square in relativistic heavy ion collisions. If all soft gluons of heavy ion beams are stopped to form the hot dense medium, the final-state interaction between the slowly expanding or almost stationary medium and the fast moving heavy quark pair (or a quarkonium) of transverse momentum $q_{T}$ is unlikely to broaden the $q_{T}$ spectrum. Instead, the final-state interaction is likely to slow down the heavy quarks due to the energy loss [23], and could reduce the averaged transverse momentum $\left\langle q_{T}^{2}\right\rangle$ [24]. On the other hand, the initial-state interaction should behave very similar to that in hadron-nucleus collision up to a possible difference in the medium density. In order to separate the effect of final-state interaction from that of initial-state interaction, and independently study the initial-state interaction and extract the medium density, we calculate the broadening of $Z$ (as well as $W$ ) bosons in relativistic heavy ion collisions at the Large Hadron Collider (LHC). Since there is effectively no final-state interaction for the production of $Z$ (or $W$ ) bosons extracted from their leptonic decay, the broadening is an ideal probe of the density of nuclear medium in the early stage of the collision [25]. If one would be able to reconstruct $Z$ (or $W$ ) bosons from their hadronic decay, the final-state interaction between the decaying jets and the medium could lead to an apparent mass shift for the vector boson and provide additional tools to extract the medium's properties.

The rest of our paper is organized as follows. In Sec. [II we review the perturbative QCD calculation for the transverse momentum broadening in Drell-Yan production to set up the notation and terminology. In Sec. III, we derive the transverse momentum broadening of heavy quarkonium production in hadron-nucleus collision. We calculate both initial-state and final-state multiple scattering. We evaluate the transverse momentum broadening in both NRQCD model and Color Evaporation model. We also discuss the broadening of heavy quarkonium production in nucleus-nucleus collisions. In Sec. IV] we calculate the broadening of $Z$ (as well as $W$ ) boson production in both hadron-nucleus and nucleus-nucleus collisions at the LHC. We argue that the transverse momentum broadening of $Z$ (or $W$ ) bosons that are reconstructed from their leptonic decay channels is an excellent probe for initial-state rescattering and the medium density at an early stage of relativistic heavy ion collisions. We present our numerical study of the transverse momentum broadening of vector boson production in Sec. $\mathrm{V}$. We discuss the extrapolation of the non-perturbative matrix elements from the fixed target energies to collider energies. We compare our calculations with data from both fixed-target experiments at Fermilab, and collider experiments at RHIC. We also predict the broadening at the LHC energy. Finally, in Sec. VI, we conclude with a summary that suggests directions for future work.

\section{TRANSVERSE MOMENTUM BROADENING IN DRELL-YAN PRODUCTION}

The Drell-Yan production of a massive pair of leptons in hadronic collision has been a potent probe of shortdistance dynamics in strong and electroweak interactions. The lack of interaction between the produced leptons and the hadronic matter makes the Drell-Yan massive dilepton production in hadron-nucleus and nucleus-nucleus collisions an ideal hard probe of initial-state partonic scattering in nuclear matter. At the fixed target energies, the Drell-Yan process is dominated by the production of a virtual photon of invariant mass $Q$ that decays into the measured lepton pairs, while the production is dominated by the $Z$ boson at the LHC energies. In nuclear collisions, it is very likely that the energetic incoming parton can have several scatterings with soft partons inside the nuclear matter before the hard collision to produce the vector boson. Such initial-state multiple scattering could induce more soft radiation from the incoming parton and broaden the parton's transverse momentum. The additional parton transverse momentum at the hard collision leads to the broadening of the observed vector bosons. 
The transverse momentum distribution of the Drell-Yan vector boson production at high $q_{T}$ is calculable in perturbative QCD [26, 27]. But, the distribution at low $q_{T} \ll Q$, which is sensitive to the soft rescattering, is not perturbatively calculable unless the resummation of large Sudakov logarithms dominates the shape of the low $q_{T}$ spectrum [10, 28, 29, 30]. Each soft rescattering can only change the incoming parton's transverse momentum by the amount close to typical virtuality of partons inside the nuclear matter, and is too soft to be perturbatively calculable. Furthermore, the potential interference between the rescattering and the parton shower, which is responsible for the large contribution of Sudakov logarithms at low $q_{T}$, could complicate the resummation of the logarithms and lead to even less control on the low $q_{T}$ spectrum. On the other hand, the averaged transverse momentum square, $\left\langle q_{T}^{2}\right\rangle$ defined in Eq. (1), is much more inclusive. If we integrate over all kinematically allowed $q_{T}$, the $\left\langle q_{T}^{2}\right\rangle$ depends on only one single hard scale, $Q$, the mass of the vector boson and is perturbatively calculable. The large logarithmic contribution to the $q_{T}^{2}$-distribution from the power of $\ln \left(Q^{2} / q_{T}^{2}\right)$ is suppressed by the $q_{T}^{2}$ weight.

The transverse momentum broadening, $\left.\Delta\left\langle q_{T}^{2}\right\rangle \equiv\left\langle q_{T}^{2}\right\rangle\right|_{A B}-\left.\left\langle q_{T}^{2}\right\rangle\right|_{h h}$, which sums over the accumulative effect of many soft rescattering, is expressed in terms of the difference of two inclusive and perturbatively calculable quantities, and is therefore calculable in perturbative QCD [7, 9]. The broadening of the Drell-Yan production of lepton pairs in hadron-nucleus collisions was first studied in terms of a non-relativisitic QED model in Ref. [12]. It was shown that initial-state interactions lead to an increase in the average of the Drell-Yan dilepton's transverse momentum square and the increase is proportional to the length of the nuclear target. The Drell-Yan transverse momentum broadening was also systematically studied in terms of perturbative QCD collinear factorization approach in a covariant gauge [13] and was further studied in Ref. [14] in a light-cone gauge. Since we calculate the transverse momentum broadening of the heavy quarkonium production in a covariant gauge in this paper, we briefly review the perturbative QCD collinear factorization approach and the covariant gauge derivation of the Drell-Yan broadening in the rest of this section.

The cross section for the Drell-Yan process in hadron-nucleus collisions, $h\left(p^{\prime}\right)+A(p) \rightarrow \gamma^{*}(q)\left[\rightarrow l^{+} l^{-}\right]+X$, where $q, p^{\prime}, p$ are the four momentum of the virtual photon, the incoming hadron, and the nucleus (per nucleon) with atomic weight $A$, respectively, can be expanded in terms of contributions with different number of rescattering,

$$
\sigma_{h A}=\sigma_{h A}^{S}+\sigma_{h A}^{D}+\ldots
$$

with superscript $S$ for single scattering, $D$ for double scattering, and etc. A single hard scattering is localized in space and time, and is unlikely to provide the target length (or the $A^{1 / 3}$-type nuclear size) enhancement to the cross section, although it can get a weaker nuclear dependence to the cross section from nuclear parton distributions [7]. The leading contribution to the broadening of the dilepton's transverse momentum square comes from the double scattering [13],

$$
\Delta\left\langle q_{T}^{2}\right\rangle_{\mathrm{DY}} \approx \int d q_{T}^{2} q_{T}^{2} \frac{d \sigma_{h A}^{D}}{d Q^{2} d q_{T}^{2}} / \frac{d \sigma_{h A}}{d Q^{2}}
$$

with the inclusive Drell-Yan cross section given by

$$
\frac{d \sigma_{h A}}{d Q^{2}} \approx \frac{d \sigma_{h A}^{S}}{d Q^{2}} \approx A \sum_{q} \int d x^{\prime} \phi_{\bar{q} / h}\left(x^{\prime}\right) \int d x \phi_{q / A}(x) \frac{d \hat{\sigma}_{q \bar{q}}}{d Q^{2}}
$$

where $A$ is the atomic weight of the nucleus, $\sum_{q}$ runs over all quark and antiquark flavors, $\phi_{\bar{q} / h}$ and $\phi_{q / A}$ represent the hadron and nuclear partonic distribution functions, respectively, and $d \hat{\sigma}_{q \bar{q}} / d Q^{2}$ is the lowest partonic $q \bar{q}$ annihilation cross section to a lepton pair of invariant mass $Q$. In Eq. (4) and the rest of this paper, we suppress all dependence on the factorization and renormalization scales. In Fig. 1] we sketch the leading order Feynman diagram that contributes to the double scattering cross section, $d \sigma_{h A}^{D}$. As shown in Fig. 1, an antiquark of momentum $x^{\prime} p^{\prime}$ from the incoming hadron scatters off a gluon from the nucleus (indicated by the bottom blob) before it annihilates with a quark from the nucleus to form a vector boson of large invariant mass, $Q$, which then decays into a lepton pair. The interference diagrams, that have both gluons in the same side of the final-state cut (the dashed line), do not contribute to the broadening in a covariant gauge calculation [13], while they are very important in the light-cone gauge calculation [14]. It is clear from the diagram that the momentum of the observed vector boson is only sensitive to the total momentum from the nucleus, which is equal to a sum of the gluon and quark momentum. Therefore, the gluon (or quark) momentum in the scattering amplitude (the left of the dashed line) is not necessary to be equal to the gluon (or quark) momentum on the right of the final-state cut. This is a consequence of the fact that there could be an arbitrary momentum flow from the nucleus through the quark line, the internal antiquark line, and back to the nucleus from the gluon line without changing both initial- and final-state. To drive the double scattering contribution to the cross section, we need to integrate over this loop momentum for both the amplitude and complex conjugate of the amplitude, or equivalently, the momentum flows through those two gluons in Fig. 1. The internal antiquark propagator following the gluon rescattering can be very large if the gluon momentum is very soft, and it can actually 
diverge if the gluon momentum vanishes. But, it is easy to verify that the singularity of the internal antiquark propagator when gluon momentum vanishes is not pinched. The integration of the gluon momentum can be deformed far away from the on-shell singularity into a perturbative off-shell region at the order of the hard scale $Q$; and the net result from the integration is given by the residue of the pole of the antiquark propagator [7, 8].

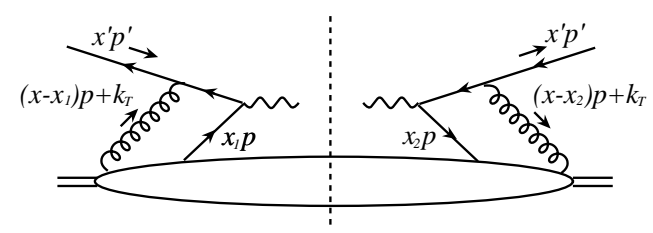

FIG. 1: Lowest order double scattering Feynman diagram that contributes to the broadening of Drell-Yan transverse momentum distribution, which shows an antiquark of momentum $x^{\prime} p^{\prime}$ of incoming hadron scatters off a gluon of a nucleus (the bottom blob) before it annihilates a quark to produce a vector boson.

Following the derivation in Ref. [13], the contribution from the double scattering diagram in Fig. 1 to the $q_{T}^{2}$-moment of Drell-Yan cross section can be expressed as

$$
\begin{aligned}
\int d q_{T}^{2} q_{T}^{2} \frac{d \sigma_{h A}^{D}}{d Q^{2} d q_{T}^{2}} & =\sum_{q} \int d q_{T}^{2} q_{T}^{2} \int d x^{\prime} \phi_{\bar{q} / h}\left(x^{\prime}\right) \int d x d x_{1} d x_{2} d^{2} k_{T} \bar{T}_{A q}\left(x, x_{1}, x_{2}, k_{T}, p\right) \\
& \times \bar{H}\left(x, x_{1}, x_{2}, k_{T}, p, q, x^{\prime} p^{\prime}\right) \delta\left(q_{T}^{2}-k_{T}^{2}\right)
\end{aligned}
$$

where the matrix element $\bar{T}_{A q}$ is given by the bottom blob in Fig. 1, which includes the propagators of all quarks and gluons connecting to the blob,

$$
\begin{aligned}
\bar{T}_{A q}\left(x, x_{1}, x_{2}, k_{T}, p\right)=\int \frac{d y^{-}}{2 \pi} \frac{d y_{1}^{-}}{2 \pi} \frac{d y_{2}^{-}}{2 \pi} \int & \frac{d^{2} y_{T}}{(2 \pi)^{2}} e^{i x_{1} p^{+} y_{1}^{-}} e^{i\left(x-x_{1}\right) p^{+} y^{-}} e^{-i\left(x-x_{2}\right) p^{+} y_{2}^{-}} e^{-i k_{T} \cdot y_{T}} \\
& \times \frac{1}{2}\left\langle p_{A}\left|A^{+}\left(y_{2}^{-}, 0_{T}\right) \bar{\psi}(0) \gamma^{+} \psi\left(y_{1}^{-}\right) A^{+}\left(y^{-}, y_{T}\right)\right| p_{A}\right\rangle
\end{aligned}
$$

where the subscript " $A q$ " indicates that the matrix element is made of the gluon and quark field operators. The partonic part $\bar{H}$ in Eq. (5) is given by the top partonic part of the diagram in Fig. 1 with two antiquark lines traced with $\left(\gamma \cdot p^{\prime}\right) / 2$, two quark lines from the nucleus traced with $(\gamma \cdot p) / 2$, and the Lorentz indices of two gluon lines from the nucleus contracted by $p^{\alpha} p^{\beta}[13]$.

The separation of the partonic part $\bar{H}$ from the hadronic matrix element $\bar{T}_{A q}$ in Eq. (5) is not yet a gauge invariant factorization. The matrix element $\bar{T}_{A q}$ in Eq. (6) has an explicit dependence on the gluon field operator $A^{+}$, which is not gauge covariant. That is, the matrix element $\bar{T}_{A q}$ cannot be made gauge invariant by the insertion of ordered gauge links between the field operators [31]. To achieve a gauge invariant factorization, it is necessary to convert the gluon field operator $A^{+}$in the matrix element into corresponding gluon field strength, $F^{+\alpha}$, with a transversely polarized Lorentz index $\alpha$. We can achieve this conversion in a covariant gauge as follows. We first expand the $k_{T}$ in the partonic part, $\bar{H}$, around $k_{T}^{2}=0$, because $k_{T}^{2} \ll Q^{2}$, and keep the first nonvanishing term, $H\left(x, x_{1}, x_{2}, p, q, x^{\prime} p^{\prime}\right)=\bar{H}\left(x, x_{1}, x_{2}, k_{T}=0, p, q, x^{\prime} p^{\prime}\right)$, which is given by the diagram in Fig. 2 . We then write the transverse momentum square, $q_{T}^{2}$ in Eq. (15) as $k_{T}^{2}$ by taking advantage of the $\delta\left(q_{T}^{2}-k_{T}^{2}\right)$, and convert $k_{T}^{2} A^{+}\left(y_{2}^{-}, 0_{T}\right) A^{+}\left(y^{-}, y_{T}\right)$ to $F_{\alpha}+\left(y_{2}^{-}, 0_{T}\right) F^{+\alpha}\left(y^{-}, y_{T}\right)$ by a partial integration [13]. Finally, upto the power corrections in $\left\langle k_{T}^{2}\right\rangle / Q^{2}$, we can rewrite the $q_{T}^{2}$-moment in Eq. (15) as

$$
\int d q_{T}^{2} q_{T}^{2} \frac{d \sigma_{h A}^{D}}{d Q^{2} d q_{T}^{2}}=\sum_{q} \int d x^{\prime} \phi_{\bar{q} / h}\left(x^{\prime}\right) \int d x d x_{1} d x_{2} T_{F q}^{(I)}\left(x, x_{1}, x_{2}, p\right) H\left(x, x_{1}, x_{2}, p, q, x^{\prime} p^{\prime}\right)
$$

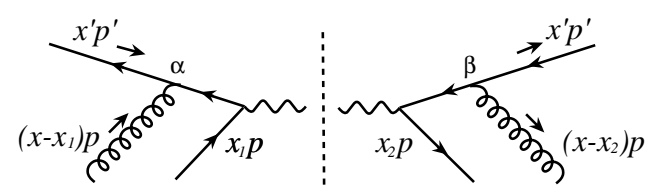

FIG. 2: Lowest-order double scattering diagram that leads to the factorized partonic part, $H$, in Eq. (9). 
where $T_{F q}$ is a twist-4 parton correlation function defined as

$$
\begin{aligned}
T_{F q}^{(I)}\left(x, x_{1}, x_{2}, p\right)=\int \frac{d y^{-}}{2 \pi} & \frac{d y_{1}^{-}}{2 \pi} \frac{d y_{2}^{-}}{2 \pi} e^{i x_{1} p^{+} y_{1}^{-}} e^{i\left(x-x_{1}\right) p^{+} y^{-}} e^{-i\left(x-x_{2}\right) p^{+} y_{2}^{-}} \\
& \times \frac{1}{2}\left\langle p_{A}\left|F_{\alpha}^{+}\left(y_{2}^{-}\right) \bar{\psi}(0) \gamma^{+} \psi\left(y_{1}^{-}\right) F^{+\alpha}\left(y^{-}\right)\right| p_{A}\right\rangle,
\end{aligned}
$$

with the superscript " $(I)$ " indicates the matrix element corresponding to the initial-state rescattering [13]. The leading order contribution to the partonic hard part from the diagram in Fig. 2] is [13]

$$
H\left(x, x_{1}, x_{2}, p, q, x^{\prime} p^{\prime}\right)=\frac{8 \pi^{2} \alpha_{s}}{N_{c}^{2}-1} C_{F}\left[\frac{1}{2 \pi} \frac{1}{x_{1}-x-i \epsilon} \frac{1}{x_{2}-x+i \epsilon}\right] \frac{d \hat{\sigma}_{q \bar{q}}}{d Q^{2}},
$$

where two unpinched poles are from the two antiquark propagators in Fig. 2. Substituting Eq. (9) to Eq. (7), and integrating over $x_{1}, x_{2}$, by taking the residues of the unpinched poles (the leading pole approximation [7]), we obtain

$$
\int d q_{T}^{2} q_{T}^{2} \frac{d \sigma_{h A}^{D}}{d Q^{2} d q_{T}^{2}}=\sum_{q} \int d x^{\prime} \phi_{\bar{q} / h}\left(x^{\prime}\right) \int d x T_{q / A}^{(I)}(x) \frac{d \hat{\sigma}_{q \bar{q}}}{d Q^{2}}\left(\frac{8 \pi^{2} \alpha_{s}}{N_{c}^{2}-1} C_{F}\right),
$$

with the measurable twist-4 quark-gluon correlation function [7, 13],

$$
\begin{aligned}
T_{q / A}^{(I)}(x)= & \int \frac{d y^{-}}{2 \pi} e^{i x p^{+} y^{-}} \int \frac{d y_{1}^{-} d y_{2}^{-}}{2 \pi} \theta\left(y^{-}-y_{1}^{-}\right) \theta\left(-y_{2}^{-}\right) \\
& \times \frac{1}{2}\left\langle p_{A}\left|F_{\alpha}^{+}\left(y_{2}^{-}\right) \bar{\psi}_{q}(0) \gamma^{+} \psi_{q}\left(y^{-}\right) F^{+\alpha}\left(y_{1}^{-}\right)\right| p_{A}\right\rangle,
\end{aligned}
$$

where the superscript "( $I)$ " again indicates the initial-state rescattering. From Eq. (3), we obtain the leading double scattering contribution to the Drell-Yan broadening [13],

$$
\Delta\left\langle q_{T}^{2}\right\rangle_{\mathrm{DY}} \approx \frac{\sum_{q} \int d x^{\prime} \phi_{\bar{q} / h}\left(x^{\prime}\right) \int d x T_{q / A}^{(I)}(x) \frac{d \hat{\sigma}_{q \bar{q}}}{d Q^{2}}\left(\frac{8 \pi^{2} \alpha_{s}}{N_{c}^{2}-1} C_{F}\right)}{A \sum_{q} \int d x^{\prime} \phi_{\bar{q} / h}\left(x^{\prime}\right) \int d x \phi_{q / A}(x) \frac{d \hat{\sigma}_{q \bar{q}}}{d Q^{2}}} .
$$

By using the model proposed for the twist-4 parton correlation functions [8, 13]

$$
T_{q / A}^{(I)}(x)=\lambda^{2} A^{4 / 3} \phi_{q / A}(x),
$$

we can express the Drell-Yan broadening in a much simpler form [13]

$$
\Delta\left\langle q_{T}^{2}\right\rangle_{\mathrm{DY}}=C_{F}\left(\frac{8 \pi^{2} \alpha_{s}}{N_{c}^{2}-1} \lambda^{2} A^{1 / 3}\right)
$$

with an unknown non-perturbative parameter $\lambda^{2}$ defined in Eq. (13). The leading contribution to the Drell-Yan broadening in Eq. (14) shows a clear $A^{1 / 3}$-type dependence and is proportional to the color factor $C_{F}$ from the rescattering between an antiquark (or a quark) and a gluon.

\section{TRANSVERSE MOMENTUM BROADENING IN HEAVY QUARKONIUM PRODUCTION}

In this section we use the same technique reviewed in last section to calculate the transverse momentum broadening of heavy quarkonium production in both hadron-nucleus and nucleus-nucleus collisions.

The heavy quarkonium's transverse momentum broadening in hadron-nucleus collision was often attributed to the initial-state multiple scattering between the active parton of the projectile and soft partons of the nuclear target before the hard collision to produce the heavy quark pair [16]. Calculation of such initial-state rescattering should be very similar to that for the Drell-Yan broadening, except that the quark-antiquark annihilation is accompanied by a much larger gluon-gluon fusion subprocess. If one considers only the gluon-gluon fusion subprocess, one should expect to have Eq. (14) for the heavy quarkonium broadening with the overall color factor $C_{F}$ replaced by $C_{A}=N_{c}=3$ due to the difference in color factors between gluon rescattering and quark rescattering. The initial-state rescattering alone 
leads to the naive expectation for the ratio of broadening between heavy quarkonium and Drell-Yan as $C_{A} / C_{F}=2.25$, which is much smaller than the data [15].

However, as discussed in the Introduction of this paper, the net broadening of heavy quarkonium's transverse momentum in hadron-nucleus collision is a combined effect of the initial-state interaction and final-state rescattering between the produced heavy quark pair and the nuclear matter. This is because a heavy quarkonium is very unlikely to form at the same time when the heavy quark pair was produced. Since the final-state rescattering is sensitive to the detailed dynamics that transmutes a heavy quark pair into a bound quarkonium, we calculate the final-state contribution to heavy quarkonium broadening in both NRQCD and Color Evaporation models.

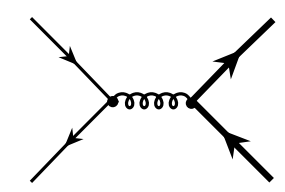

(a)
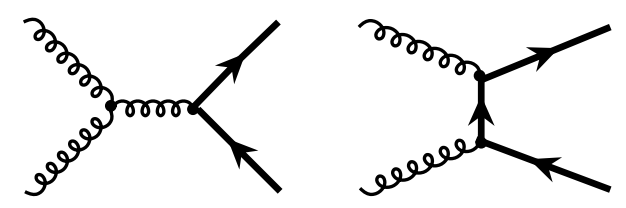

(b)

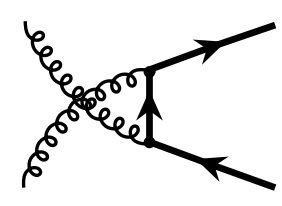

FIG. 3: Lowest order Feynman diagram for light quark-antiquark annihilation (a) and for gluon-gluon fusion to a pair of heavy quark.

\section{A. Color Evaporation Model}

In the Color Evaporation model (CEM), heavy quarkonium production is factorized into two steps: the production of a pair of heavy quarks with an invariant mass $Q$ followed by a non-perturbative hadronization process with an universal transition probability for the pair to become a bound quarkonium [20]. It was assumed that the transition probability is the same for all heavy quark pairs whose invariant mass is less than the mass threshold of producing two open flavor heavy mesons, and the cross section for producing a heavy quarkonium, $H$, can be written as [20]

$$
\sigma_{h A \rightarrow H}^{\mathrm{CEM}}=F_{Q \bar{Q} \rightarrow H} \int_{4 m_{Q}^{2}}^{4 M_{Q}^{2}} d Q^{2} \frac{d \sigma_{h A \rightarrow Q \bar{Q}}}{d Q^{2}},
$$

where $F_{Q \bar{Q} \rightarrow H}$ is a non-perturbative transition probability and is independent of the color and angular momentum of the heavy quark pair. There is one transition probability for each heavy quarkonium state, $H$. In Eq. (15), the inclusive cross section for producing a pair of heavy quarks of invariant mass $Q$ can be factorized as [35]

$$
\frac{d \sigma_{h A \rightarrow Q \bar{Q}}}{d Q^{2}}=A \sum_{a, b} \int d x^{\prime} \phi_{a / h}\left(x^{\prime}\right) \int d x \phi_{b / A}(x) \frac{d \hat{\sigma}_{a b \rightarrow Q \bar{Q}}}{d Q^{2}},
$$

where $\sum_{a, b}$ sum over all parton flavors, and $d \hat{\sigma}_{a b \rightarrow Q \bar{Q}} / d Q^{2}$ is a short-distance hard part for two partons of flavor $a$ and $b$ to produce a pair of heavy quarks of invariant mass $Q$. At the lowest order, they are given by the light quark-antiquark annihilation and gluon-gluon fusion subprocess, as sketched in Fig. 3. The transition probability in Eq. (15) is assumed to be universal and independent of how the heavy quark pair was produced. It fixes the overall normalization for the cross section of heavy quarkonium production in different collision processes and provides the predictive power of the model. The model has been reasonably successful when comparing with data of inclusive heavy quarkonium production [32, $\underline{33}$.

Similar to Fig. 2] the leading order double scattering diagrams for producing a heavy quark pair are sketched in Fig. 4 for quark-antiquark annihilation subprocess, and in Fig. 5 for gluon-gluon fusion subprocess, respectively. The

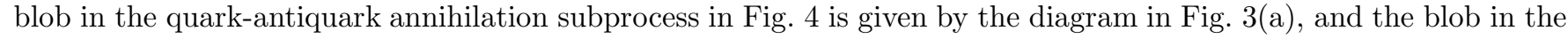
gluon-gluon fusion subprocess in Fig. [5 is given by a sum of the three diagrams in Fig. [3(b).

In CEM, the transverse momentum broadening of a heavy quarkonium is equal to the transverse momentum broadening of the parent heavy quark pair, since the transition probability from a heavy quark pair to a bound quarkonium is given by a constant, $F_{Q \bar{Q} \rightarrow H}$. We use the same method reviewed in last section to calculate the 


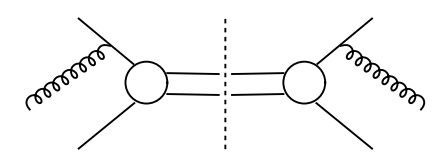

(a)

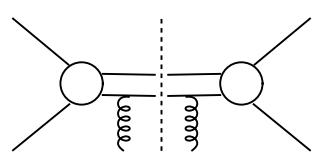

(b)

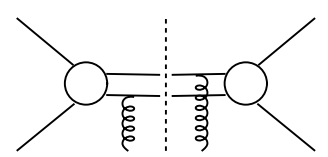

(c)

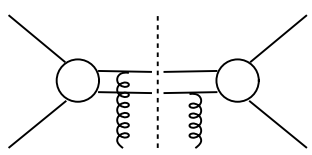

(d)

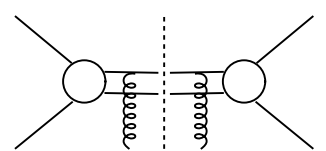

(e)

FIG. 4: Leading order double scattering diagrams for $q \bar{q} \rightarrow Q \bar{Q}$ : initial-state double scattering (a), and final-state double scattering (b), (c), (d), and (e).

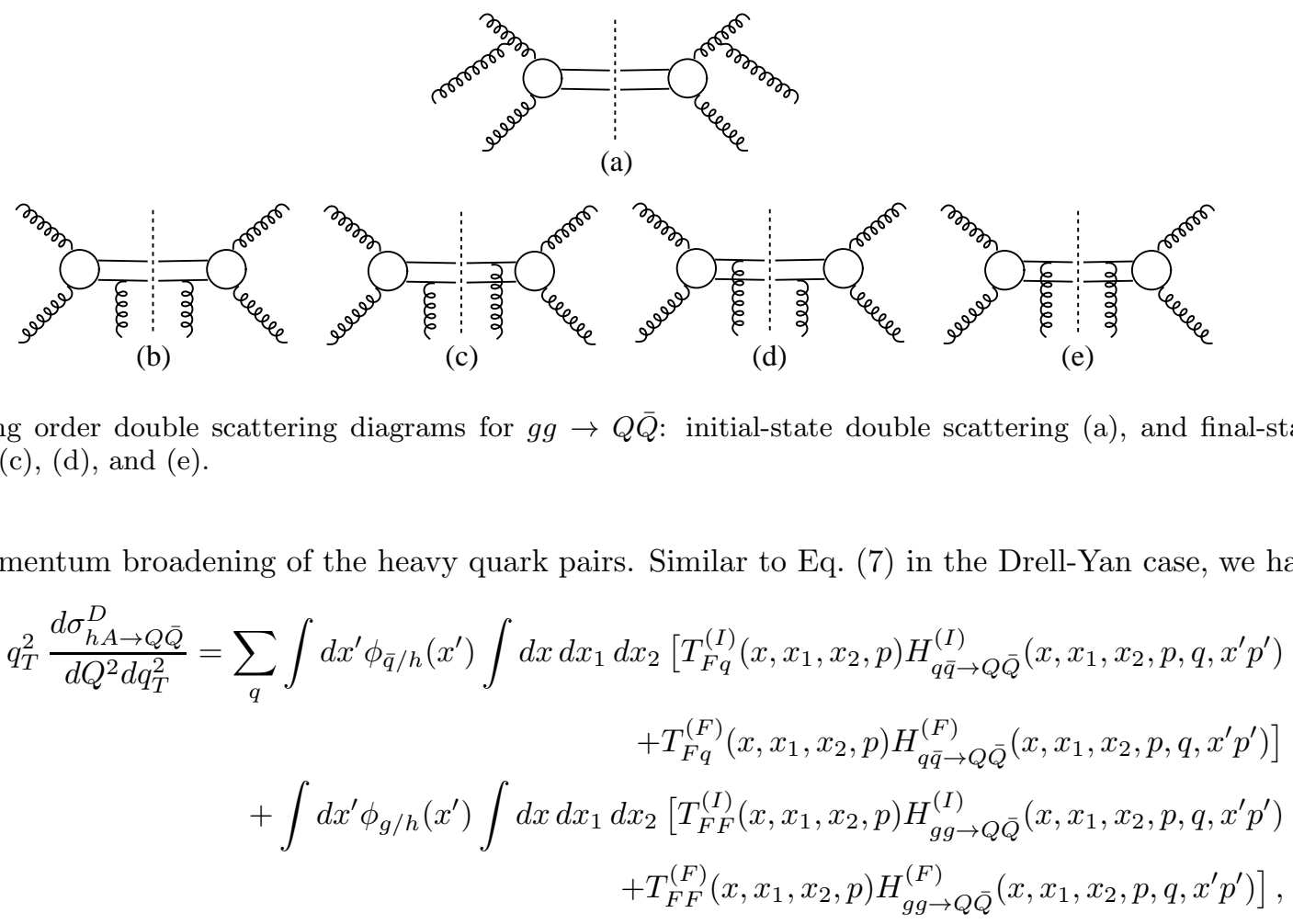

where the superscripts, "(I)" and "(F)", indicate the initial-state and final-state rescattering, respectively, and the matrix element $T_{F q}^{(I)}$ is given in Eq. (8).$T_{F F}^{(I)}$ is given by

$$
\begin{aligned}
T_{F F}^{(I)}\left(x, x_{1}, x_{2}, p\right)=\int \frac{d y^{-}}{2 \pi} & \frac{d y_{1}^{-}}{2 \pi} \frac{d y_{2}^{-}}{2 \pi} e^{i x_{1} p^{+} y_{1}^{-}} e^{i\left(x-x_{1}\right) p^{+} y^{-}} e^{-i\left(x-x_{2}\right) p^{+} y_{2}^{-}} \\
& \times\left\langle p_{A}\left|F_{\alpha}^{+}\left(y_{2}^{-}\right) F^{\sigma+}(0) F_{\sigma}^{+}\left(y_{1}^{-}\right) F^{+\alpha}\left(y^{-}\right)\right| p_{A}\right\rangle .
\end{aligned}
$$

The matrix elements with final-state rescattering, $T_{F q}^{(F)}$ and $T_{F F}^{(F)}$, have the same expressions as corresponding matrix elements with initial-state rescattering, since the field operators in the definition of the multiparton matrix elements in the collinear factorization approach commute on the light-cone [31].

The partonic parts, $H_{q \bar{q} \rightarrow Q \bar{Q}}^{(I, F)}$, are given by the Feynman diagrams in Fig. 团 with the quark line from hadron (top) traced with $\left(\gamma \cdot p^{\prime}\right) / 2$, the quark line from nucleus (bottom) traced with $(\gamma \cdot p) / 2$, and gluon lines contracted with

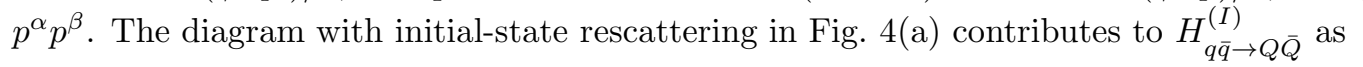

$$
H_{q \bar{q} \rightarrow Q \bar{Q}}^{(I)}=H_{q \bar{q} \rightarrow Q \bar{Q}}^{\left.(4)_{i}\right)}=\frac{8 \pi^{2} \alpha_{s}}{N_{c}^{2}-1} C_{F}\left[\frac{1}{2 \pi} \frac{1}{x_{1}-x-i \epsilon} \frac{1}{x_{2}-x+i \epsilon}\right] \frac{d \hat{\sigma}_{q \bar{q} \rightarrow Q \bar{Q}}}{d Q^{2}},
$$

where the lowest order partonic cross section from $q \bar{q}$ annihilation to a heavy quark pair of invariant mass $Q$ is given in Ref. [34]. All four diagrams with the final-state rescattering, in Figs. 4 (b), (c), (d), and (e), contribute to $H_{q \bar{q} \rightarrow Q \bar{Q}}^{(F)}$ 
as

$$
H_{q \bar{q} \rightarrow Q \bar{Q}}^{(F)}=H_{q \bar{q} \rightarrow Q \bar{Q}}^{(4 \overrightarrow{4}+4 l t+43)}=\frac{8 \pi^{2} \alpha_{s}}{N_{c}^{2}-1} C_{A}\left[\frac{1}{2 \pi} \frac{1}{x_{1}-x+i \epsilon} \frac{1}{x_{2}-x-i \epsilon}\right] \frac{d \hat{\sigma}_{q \bar{q} \rightarrow Q \bar{Q}}}{d Q^{2}}
$$

The final-state contribution in Eq. (20) is very similar to the initial-state contribution in Eq. (19) except the overall color factor and the location of the unpinched poles. The difference in the location of the pinched poles, indicated by the sign difference of the $i \epsilon$, is a consequence of the order of the rescattering taken place either before or after the hard collision. The overall color factor for the final-state interaction, $C_{A}$ in Eq. (20), indicates that as far as the color is concerned, the rescattering of a heavy quark pair is effectively the same as that of a color-octet gluon when the effect is calculated in the Color Evaporation model.

Similarly, we evaluate the double scattering diagrams from gluon-gluon fusion subprocess in Fig. [5 and obtain their contribution to the partonic hard part,

$$
H_{g g \rightarrow Q \bar{Q}}^{(I)}=H_{g g \rightarrow Q \bar{Q}}^{(5,)}=\frac{8 \pi^{2} \alpha_{s}}{N_{c}^{2}-1} C_{A}\left[\frac{1}{2 \pi} \frac{1}{x_{1}-x-i \epsilon} \frac{1}{x_{2}-x+i \epsilon}\right] \frac{d \hat{\sigma}_{g g \rightarrow Q \bar{Q}}}{d Q^{2}},
$$

for the initial-state double scattering, and

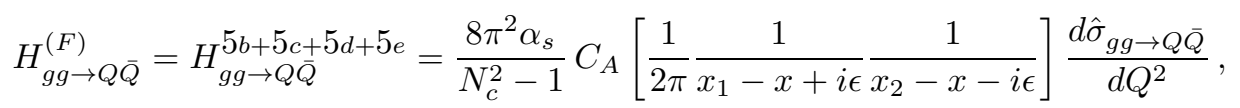

for the final-state double scattering. We find that the contribution to the gluon-gluon fusion subprocess from the final-state double scattering is the same as that from the initial-state interaction. That is because the rescattering effect of a heavy quark pair is the same as that of a color-octet gluon when the effect is calculated in the Color Evaporation model.

Substituting the partonic hard parts in Eqs. (19), (20), (21), and (22) into Eq. (17), integrating over the momentum fractions, $x_{1}$ and $x_{2}$ of the rescattering gluons under the leading pole approximation, we obtain the leading double scattering contribution to the $q_{T}^{2}$-moment of producing a heavy quark pair in hadron-nucleus collisions,

$$
\begin{aligned}
\int d q_{T}^{2} q_{T}^{2} \frac{d \sigma_{h A \rightarrow Q \bar{Q}}^{D}}{d Q^{2} d q_{T}^{2}}=\left[\frac{8 \pi^{2} \alpha_{s}}{N_{c}^{2}-1}\right] & \left(\sum_{q} \int d x^{\prime} \phi_{\bar{q} / h}\left(x^{\prime}\right) \int d x\left[C_{F} T_{q / A}^{(I)}(x)+C_{A} T_{q / A}^{(F)}(x)\right] \frac{d \hat{\sigma}_{q \bar{q} \rightarrow Q \bar{Q}}}{d Q^{2}}\right. \\
& \left.+\int d x^{\prime} \phi_{g / h}\left(x^{\prime}\right) \int d x\left[C_{A} T_{g / A}^{(I)}(x)+C_{A} T_{g / A}^{(F)}(x)\right] \frac{d \hat{\sigma}_{g g \rightarrow Q \bar{Q}}}{d Q^{2}}\right),
\end{aligned}
$$

where the quark-gluon correlation function, $T_{q / A}^{(I)}$, is given in Eq. (11), the $T_{g / A}^{(I)}$ is given by

$$
\begin{aligned}
T_{g / A}^{(I)}(x) & =\int \frac{d y^{-}}{2 \pi} e^{i x p^{+} y^{-}} \int \frac{d y_{1}^{-} d y_{2}^{-}}{2 \pi} \theta\left(y^{-}-y_{1}^{-}\right) \theta\left(-y_{2}^{-}\right) \\
& \times \frac{1}{x p^{+}}\left\langle p_{A}\left|F_{\alpha}^{+}\left(y_{2}^{-}\right) F^{\sigma+}(0) F_{\sigma}^{+}\left(y^{-}\right) F^{+\alpha}\left(y_{1}^{-}\right)\right| p_{A}\right\rangle,
\end{aligned}
$$

and $T_{q / A}^{(F)}$ and $T_{q / A}^{(F)}$ are given by the same expressions in Eq. (11) and Eq. (24), respectively, except the $\theta$-functions are replaced as [7]

$$
\theta\left(y^{-}-y_{1}^{-}\right) \theta\left(-y_{2}^{-}\right) \rightarrow \theta\left(y_{1}^{-}-y^{-}\right) \theta\left(y_{2}^{-}\right),
$$

due to the different order of rescattering (or the sign of the $i \epsilon$ for the unpinched poles).

By integrating over the invariant mass of the heavy quark pair we can define the heavy quarkonium transverse momentum broadening in CEM as

$$
\Delta\left\langle q_{T}^{2}\right\rangle_{\mathrm{HQ}}^{\mathrm{CEM}} \approx \int d q_{T}^{2} q_{T}^{2} \int_{4 m_{Q}^{2}}^{4 M_{Q}^{2}} d Q^{2} \frac{d \sigma_{h A \rightarrow Q \bar{Q}}^{D}}{d Q^{2} d q_{T}^{2}} / \int_{4 m_{Q}^{2}}^{4 M_{Q}^{2}} d Q^{2} \frac{d \sigma_{h A \rightarrow Q \bar{Q}}}{d Q^{2}},
$$

where $m_{Q}$ and $M_{Q}$ are heavy quark mass and open flavor heavy meson mass, respectively.

As pointed out in Ref. [31], the field operators on the light-cone in the definition of the multiparton matrix elements, as those in Eqs. (11) and (24) commute. The matrix element with initial-state rescattering is equal to corresponding matrix element with final-state rescattering, if the phase space interaction of these two matrix elements, such as the 
$\int d y^{-} d y_{1}^{-} d y_{2}^{-} \theta\left(y^{-}-y_{1}^{-}\right) \theta\left(-y_{2}^{-}\right)$in Eq. (11) for initial-state rescattering and $\int d y^{-} d y_{1}^{-} d y_{2}^{-} \theta\left(y_{1}^{-}-y^{-}\right) \theta\left(y_{2}^{-}\right)$for the corresponding final-state rescattering, are the same [13]. However, the phase space integration for the final-state interaction in heavy quarkonium production may not cover the full size of the nuclear medium if the heavy quark pair becomes a physical quarkonium or transmutes into a color singlet pre-hadron quarkonium state before the pair exits the nuclear medium. Rescattering between a quarkonium and nuclear medium and that between a colored heavy quark pair and the same medium could be different, and lead to a different heavy quarkonium broadening.

It was argued in Ref. [18] that a physical quarkonium state is likely to form outside nuclear matter in hadronnucleus collision. Therefore, the matrix elements with final- and initial-state rescattering could have the same phase space interaction. If we assume that the matrix elements with the final- and initial-state rescattering are the same, $T_{q / A}^{(F)}(x)=T_{q / A}^{(I)}(x)$ and $T_{g / A}^{(F)}(x)=T_{g / A}^{(I)}(x)$, and assume the same model for both twist-4 quark-gluon and gluon-gluon correlation functions,

$$
T_{g / A}^{(I)}(x)=\lambda^{2} A^{4 / 3} \phi_{g / A}(x),
$$

we can express the heavy quarkonium broadening in hadron-nucleus collisions as,

$$
\Delta\left\langle q_{T}^{2}\right\rangle_{\mathrm{HQ}}^{\mathrm{CEM}}=\left(\frac{8 \pi^{2} \alpha_{s}}{N_{c}^{2}-1} \lambda^{2} A^{1 / 3}\right) \frac{\left(C_{F}+C_{A}\right) \sigma_{q \bar{q}}+2 C_{A} \sigma_{g g}}{\sigma_{q \bar{q}}+\sigma_{g g}},
$$

where $\sigma_{q \bar{q}}$ and $\sigma_{g g}$ are the lowest order inclusive cross sections from the $q \bar{q} \rightarrow Q \bar{Q} \rightarrow H$ and $g g \rightarrow Q \bar{Q} \rightarrow H$ subprocess, respectively. They are given by [5, 34]

$$
\begin{aligned}
& \sigma_{q \bar{q}}=F_{Q \bar{Q} \rightarrow H} \int_{4 m_{Q}^{2}}^{4 M_{Q}^{2}} d Q^{2} \sum_{q} \int d x^{\prime} \phi_{\bar{q} / h}\left(x^{\prime}\right) \int d x \phi_{q / A}(x) \frac{d \hat{\sigma}_{q \bar{q} \rightarrow Q \bar{Q}}}{d Q^{2}} \\
& \sigma_{g g}=F_{Q \bar{Q} \rightarrow H} \int_{4 m_{Q}^{2}}^{4 M_{Q}^{2}} d Q^{2} \int d x^{\prime} \phi_{g / h}\left(x^{\prime}\right) \int d x \phi_{g / A}(x) \frac{d \hat{\sigma}_{g g \rightarrow Q \bar{Q}}}{d Q^{2}},
\end{aligned}
$$

where the lowest order partonic cross sections to produce a pair of $Q \bar{Q}$ are given in Ref. [34]. If the gluon-gluon subprocess dominates the heavy quarkonium production rate, $\sigma_{g g} \gg \sigma_{q \bar{q}}$, we have

$$
\Delta\left\langle q_{T}^{2}\right\rangle_{\mathrm{HQ}}^{\mathrm{CEM}} \approx 2 C_{A}\left(\frac{8 \pi^{2} \alpha_{s}}{N_{c}^{2}-1} \lambda^{2} A^{1 / 3}\right)
$$

By comparing the Drell-Yan broadening in Eq. (14) and the leading heavy quarkonium broadening in Eq. (31), we conclude that the leading contribution to heavy quarkonium transverse momentum broadening in hadron-nucleus collision, calculated in CEM, is about $2 C_{A} / C_{F}=4.5$ times Drell-Yan broadening.

\section{B. Non-Relativistic QCD Model}

The application of NRQCD to the production of a heavy quarkonium $H$ in hadronic collisions relies on the proposed factorization formalism [19],

$$
\sigma_{h A \rightarrow H}^{\mathrm{NRQCD}}=A \sum_{a, b} \int d x^{\prime} \phi_{a / h}\left(x^{\prime}\right) \int d x \phi_{b / A}(x)\left[\sum_{n} H_{a b \rightarrow Q \bar{Q}[n]}\left\langle\mathcal{O}^{H}(n)\right\rangle\right]
$$

where $a$ and $b$ run over all parton flavors, and $H_{a b \rightarrow Q \bar{Q}[n]}$ are perturbatively calculable coefficient functions for producing the heavy $Q \bar{Q}[n]$ states. The state of the heavy quark pair, $[n]$, is characterized by the pair's rotational, ${ }^{2 s+1} L_{J}$, and color quantum numbers. The coefficient function for producing each $Q \bar{Q}[n]$ state is perturbatively calculable in a power series of the strong coupling constant, $\alpha_{s}$. The matrix elements of $\left\langle\mathcal{O}^{H}(n)\right\rangle$ in Eq. (32) describe the nonperturbative hadronization dynamics and give the probability for the pair to become a physical heavy quarkonium $H$ [19, 36]. The matrix elements should be universal. That is necessary for the predictive power of the NRQCD formalism. The expansion in Eq. (32) is organized according to the effective power of the heavy quark pair's relative velocity. Although it still lacks a fully compelling proof for the NRQCD factorization formula in Eq. (32) [36], the formalism for heavy quarkonium production has had many successes, in particular, its success in interpreting the $\mathrm{CDF}$ data on $\mathrm{J} / \psi$ and $\psi^{\prime}$ production as a function of transverse momentum [4, 37]. 
In NRQCD model of heavy quarkonium production, the transition probability from a heavy quark pair to a bound quarkonium is sensitive to the pair's rotational and color quantum numbers. Partonic multiple scattering has a potential to change not only the heavy quark pair's momentum, but also the pair's color and other quantum numbers. Therefore, the transverse momentum broadening calculated in NRQCD model is not necessary the same as that calculated in CEM in last subsection. If the difference is significant, a precise measurement of transverse momentum broadening could shed some lights on heavy quarkonium's production mechanism.

We assume that the $q_{T}^{2}$-moment of heavy quarkonium production, $\int d q_{T}^{2}\left(q_{T}^{2}\right)^{n} d \sigma_{h A \rightarrow H}^{\mathrm{NRQCD}} / d q_{T}^{2}$ with $n \geq 0$, can be factorized in the same way as the $0^{\text {th }}$-moment given in Eq. (32). We calculate the leading double scattering contribution to the broadening of the first moment. Like the calculation done in CEM in last subsection, the partonic double scattering diagrams are given in Figs. 4 and 5. Similar to Eq. (17), we obtain

$$
\begin{array}{r}
\int d q_{T}^{2} q_{T}^{2} \frac{d \sigma_{h A \rightarrow H}^{D}}{d q_{T}^{2}}=\sum_{q} \int d x^{\prime} \phi_{\bar{q} / h}\left(x^{\prime}\right) \int d x d x_{1} d x_{2}\left[T_{F q}^{(I)}\left(x, x_{1}, x_{2}, p\right) H_{q \bar{q} \rightarrow H}^{(I)}\left(x, x_{1}, x_{2}, p, q, x^{\prime} p^{\prime}\right)\right. \\
\left.+T_{F q}^{(F)}\left(x, x_{1}, x_{2}, p\right) H_{q \bar{q} \rightarrow H}^{(F)}\left(x, x_{1}, x_{2}, p, q, x^{\prime} p^{\prime}\right)\right] \\
+\int d x^{\prime} \phi_{g / h}\left(x^{\prime}\right) \int d x d x_{1} d x_{2}\left[T_{F F}^{(I)}\left(x, x_{1}, x_{2}, p\right) H_{g g \rightarrow H}^{(I)}\left(x, x_{1}, x_{2}, p, q, x^{\prime} p^{\prime}\right)\right. \\
\left.+T_{F F}^{(F)}\left(x, x_{1}, x_{2}, p\right) H_{g g \rightarrow H}^{(F)}\left(x, x_{1}, x_{2}, p, q, x^{\prime} p^{\prime}\right)\right],
\end{array}
$$

with the partonic cross sections defined as

$$
\begin{aligned}
& H_{q \bar{q} \rightarrow H}^{(I, F)}\left(x, x_{1}, x_{2}, p, q, x^{\prime} p^{\prime}\right)=\sum_{n} H_{q \bar{q}}^{(I, F)}(n)\left\langle\mathcal{O}^{H}(n)\right\rangle, \\
& H_{g g \rightarrow H}^{(I, F)}\left(x, x_{1}, x_{2}, p, q, x^{\prime} p^{\prime}\right)=\sum_{n} H_{g g}^{(I, F)}(n)\left\langle\mathcal{O}^{H}(n)\right\rangle,
\end{aligned}
$$

where $\sum_{n}$ sums over all possible $Q \bar{Q}$ states, $n$, with appropriate spin and color quantum numbers [38], and $H_{q \bar{q}}^{(I, F)}(n)$ and $H_{g g}^{(I, F)}(n)$, whose dependence on parton momentum fractions and kinematic variables are suppressed, represent partonic hard parts for producing a heavy quark pair at a quantum state $n$ from quark-antiquark annihilation subprocess and gluon-gluon fusion subprocess, respectively.

The partonic parts for the quark-antiquark annihilation subprocess, $H_{q \bar{q}}^{(I, F)}(n)$ in Eq. (34), are derived from diagrams

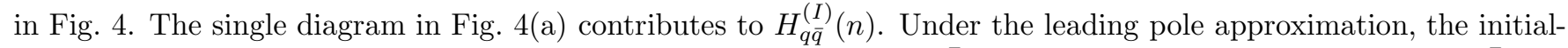
state rescattering does not change the nature of the $s$-channel $q \bar{q} \rightarrow Q \bar{Q}$ subprocess, which produces a heavy $Q \bar{Q}$ pair in a color octet and spin-1 state: $n={ }^{3} S_{1}^{(8)}$. The corresponding hard part is given by

$$
H_{q \bar{q}}^{(I)}\left({ }^{3} S_{1}^{(8)}\right)=\frac{8 \pi^{2} \alpha_{s}}{N_{c}^{2}-1} C_{F}\left[\frac{1}{2 \pi} \frac{1}{x_{1}-x-i \epsilon} \frac{1}{x_{2}-x+i \epsilon}\right] H_{q \bar{q}}^{(0)}\left({ }^{3} S_{1}^{(8)}\right),
$$

where $H_{q \bar{q}}^{(0)}\left({ }^{3} S_{1}^{(8)}\right)$ is the lowest order short-distance coefficient for $q \bar{q} \rightarrow Q \bar{Q}\left({ }^{3} S_{1}^{(8)}\right)$ subprocess and is given by

$$
H_{q \bar{q}}^{(0)}\left({ }^{3} S_{1}^{(8)}\right)=\frac{\pi^{3} \alpha_{s}^{2}}{M^{3}} \frac{16}{27} \delta\left(\hat{s}-M^{2}\right),
$$

with the mass of a quarkonium: $M=2 m_{Q}$. The final-state rescattering effect to the $q \bar{q}$ annihilation subprocess comes from the four diagrams in Figs. 4 (b), (c), (d), and (e). The additional gluon rescattering in the final-state allows more quantum states for the produced $Q \bar{Q}$ pair. At this order, we have the following nonvanishing states: $n={ }^{3} S_{1}^{(8)},{ }^{3} P_{J=1,2}^{(1,8)}$, and corresponding hard parts,

$$
\begin{gathered}
H_{q \bar{q}}^{(F)}\left({ }^{3} S_{1}^{(8)}\right)=\frac{8 \pi^{2} \alpha_{s}}{N_{c}^{2}-1} C_{A}\left[\frac{1}{2 \pi} \frac{1}{x_{1}-x+i \epsilon} \frac{1}{x_{2}-x-i \epsilon}\right] H_{q \bar{q}}^{(0)}\left({ }^{3} S_{1}^{(8)}\right), \\
H_{q \bar{q}}^{(F)}\left({ }^{3} P_{J=1,2}^{(1,8)}\right)=\frac{8 \pi^{2} \alpha_{s}}{N_{c}^{2}-1}\left[\frac{1}{2 \pi} \frac{1}{x_{1}-x+i \epsilon} \frac{1}{x_{2}-x-i \epsilon}\right] H_{q \bar{q}}^{(1)}\left({ }^{3} P_{J=1,2}^{(1,8)}\right),
\end{gathered}
$$

where $H_{q \bar{q}}^{(0)}\left({ }^{3} S_{1}^{(8)}\right)$ is given in Eq. (36) and the leading order $P$-wave contribution is given by

$$
\begin{aligned}
H_{q \bar{q}}^{(1)}\left({ }^{3} P_{1}^{(8)}\right) & =\frac{5}{3} H_{q \bar{q}}^{(1)}\left({ }^{3} P_{2}^{(8)}\right)=\frac{\pi^{3} \alpha_{s}^{2}}{M^{3}} \frac{40}{27} \frac{1}{3 m_{Q}^{2}} \delta\left(\hat{s}-M^{2}\right), \\
H_{q \bar{q}}^{(1)}\left({ }^{3} P_{1}^{(1)}\right) & =\frac{5}{3} H_{q \bar{q}}^{(1)}\left({ }^{3} P_{2}^{(1)}\right)=\frac{\pi^{3} \alpha_{s}^{2}}{M^{3}} \frac{64}{81} \frac{1}{3 m_{Q}^{2}} \delta\left(\hat{s}-M^{2}\right) .
\end{aligned}
$$


The nonvanishing contribution to the ${ }^{3} P_{J=1,2}^{(1,8)}$ states is a consequence of the gluon rescattering, which effectively provides a $g g \rightarrow Q \bar{Q}$ subprocess.

Similarly, partonic parts for the gluon-gluon fusion subprocess, $H_{g g}^{(I, F)}(n)$ in Eq. (34), are derived from diagrams in Fig. 5. Unlike the quark-antiquark annihilation subprocess, the heavy quark pair produced in gluon-gluon subprocess can have more than one quantum state. For the initial-state rescattering diagram in Fig. 5 (a), the heavy quark pair with $n={ }^{1} S_{0}^{(1,8)},{ }^{3} P_{J=0,2}^{(1,8)}$ can all give nonvanishing contribution to $H_{g g}^{(I)}(n)$. The four final-state rescattering diagrams

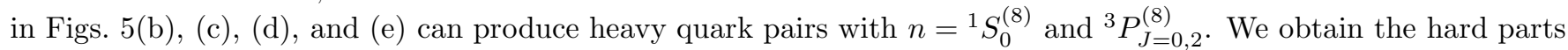
from gluon-gluon fusion diagrams in Fig. 5 as

$$
\begin{aligned}
H_{g g}^{(I)}\left({ }^{1} S_{0}^{(1,8)},{ }^{3} P_{J=0,2}^{(1,8)}\right) & =\frac{8 \pi^{2} \alpha_{s}}{N_{c}^{2}-1} C_{A}\left[\frac{1}{2 \pi} \frac{1}{x_{1}-x-i \epsilon} \frac{1}{x_{2}-x+i \epsilon}\right] H_{g g}^{(0)}\left({ }^{1} S_{0}^{(1,8)},{ }^{3} P_{J=0,2}^{(1,8)}\right), \\
H_{g g}^{(F)}\left({ }^{1} S_{0}^{(8)},{ }^{3} P_{J=0,2}^{(1,8)}\right) & =\frac{8 \pi^{2} \alpha_{s}}{N_{c}^{2}-1} C_{A}\left[\frac{1}{2 \pi} \frac{1}{x_{1}-x+i \epsilon} \frac{1}{x_{2}-x-i \epsilon}\right] H_{g g}^{(0)}\left({ }^{1} S_{0}^{(8)},{ }^{3} P_{J=0,2}^{(8)}\right),
\end{aligned}
$$

where $H_{g g}^{(0)}\left({ }^{1} S_{0}^{(1,8)},{ }^{3} P_{J=0,2}^{(1,8)}\right)$ are the lowest order cross sections without the rescattering,

$$
\begin{aligned}
H_{g g}^{(0)}\left({ }^{1} S_{0}^{(1,8)}\right) & =\frac{\pi^{3} \alpha_{s}^{2}}{M^{3}} B_{n} \delta\left(\hat{s}-M^{2}\right), \\
H_{g g}^{(0)}\left({ }^{3} P_{0}^{(1,8)}\right) & =\frac{\pi^{3} \alpha_{s}^{2}}{M^{3}} B_{n} \frac{3}{m_{Q}^{2}} \delta\left(\hat{s}-M^{2}\right), \\
H_{g g}^{(0)}\left({ }^{3} P_{2}^{(1,8)}\right) & =\frac{\pi^{3} \alpha_{s}^{2}}{M^{3}} B_{n} \frac{4}{5 m_{Q}^{2}} \delta\left(\hat{s}-M^{2}\right),
\end{aligned}
$$

with a color factor $B_{n}=2 / 9$ for a color-singlet state and $5 / 12$ for a color-octet state, respectively.

Having obtained the short-distance hard parts, we can derive the leading double scattering contribution to the $q_{T}^{2}$-moment of heavy quarkonium production in Eq. (33). In this paper, we limit ourselves to the direct production of spin-1, $S$-wave heavy quarkonia, such as J/ $/ \Upsilon$ and etc. After neglecting the color singlet $Q \bar{Q}$ states not equal to ${ }^{3} S_{1}$, we are left with the following $Q \bar{Q}$ states, which contribute to the direct production of a $H\left({ }^{3} S_{1}\right)$ heavy quarkonium,

$$
\begin{aligned}
& q \bar{q} \rightarrow Q \bar{Q}\left({ }^{3} S_{1}^{(8)},{ }^{3} P_{J=1,2}^{(8)}\right) \rightarrow H\left({ }^{3} S_{1}\right), \\
& g g \rightarrow Q \bar{Q}\left({ }^{1} S_{0}^{(8)},{ }^{3} P_{J=0,2}^{(8)}\right) \rightarrow H\left({ }^{3} S_{1}\right) .
\end{aligned}
$$

From Eq. (34), we obtain by summing over all $Q \bar{Q}$ states in Eq. (42),

$$
\begin{aligned}
H_{q \bar{q} \rightarrow H}^{(I)} & =\frac{8 \pi^{2} \alpha_{s}}{N_{c}^{2}-1}\left[\frac{1}{2 \pi} \frac{1}{x_{1}-x-i \epsilon} \frac{1}{x_{2}-x+i \epsilon}\right] C_{F} H_{q \bar{q}}^{(0)}\left({ }^{3} S_{1}^{(8)}\right)\left\langle\mathcal{O}^{H}\left({ }^{3} S_{1}^{(8)}\right)\right\rangle \\
& =\frac{8 \pi^{2} \alpha_{s}}{N_{c}^{2}-1}\left[\frac{1}{2 \pi} \frac{1}{x_{1}-x-i \epsilon} \frac{1}{x_{2}-x+i \epsilon}\right] C_{F} \hat{\sigma}_{q \bar{q}}^{(0)},
\end{aligned}
$$

where the lowest order quark-antiquark annihilation cross section is defined as

$$
\hat{\sigma}_{q \bar{q}}^{(0)} \equiv H_{q \bar{q}}^{(0)}\left({ }^{3} S_{1}^{(8)}\right)\left\langle\mathcal{O}^{H}\left({ }^{3} S_{1}^{(8)}\right)\right\rangle=\frac{\pi^{3} \alpha_{s}^{2}}{M^{3}} \frac{16}{27} \delta\left(\hat{s}-M^{2}\right)\left\langle\mathcal{O}^{H}\left({ }^{3} S_{1}^{(8)}\right)\right\rangle
$$

with the nonperturbative NRQCD matrix element, $\left\langle\mathcal{O}^{H}\left({ }^{3} S_{1}^{(8)}\right)\right\rangle$, for a $Q \bar{Q}\left[{ }^{3} S_{1}^{(8)}\right]$ pair to become a heavy quarkonium $H$. Similarly, we have the final-state rescattering contribution,

$$
\begin{aligned}
H_{q \bar{q} \rightarrow H}^{(F)}=\frac{8 \pi^{2} \alpha_{s}}{N_{c}^{2}-1}\left[\frac{1}{2 \pi} \frac{1}{x_{1}-x+i \epsilon} \frac{1}{x_{2}-x-i \epsilon}\right] & \left(C_{A} H_{q \bar{q}}^{(0)}\left({ }^{3} S_{1}^{(8)}\right)\left\langle\mathcal{O}^{H}\left({ }^{3} S_{1}^{(8)}\right)\right\rangle\right. \\
& \left.+H_{q \bar{q}}^{(1)}\left({ }^{3} P_{1}^{(8)}\right)\left\langle\mathcal{O}^{H}\left({ }^{3} P_{1}^{(8)}\right)\right\rangle+H_{q \bar{q}}^{(1)}\left({ }^{3} P_{2}^{(8)}\right)\left\langle\mathcal{O}^{H}\left({ }^{3} P_{2}^{(8)}\right)\right\rangle\right) \\
= & \frac{8 \pi^{2} \alpha_{s}}{N_{c}^{2}-1}\left[\frac{1}{2 \pi} \frac{1}{x_{1}-x+i \epsilon} \frac{1}{x_{2}-x-i \epsilon}\right]\left(C_{A} \hat{\sigma}_{q \bar{q}}^{(0)}+\hat{\sigma}_{q \bar{q}}^{(1)}\right),
\end{aligned}
$$

where we defined the $P$-wave contribution as

$$
\begin{aligned}
\hat{\sigma}_{q \bar{q}}^{(1)} & \equiv H_{q \bar{q}}^{(1)}\left({ }^{3} P_{1}^{(8)}\right)\left\langle\mathcal{O}^{H}\left({ }^{3} P_{1}^{(8)}\right)\right\rangle+H_{q \bar{q}}^{(1)}\left({ }^{3} P_{2}^{(8)}\right)\left\langle\mathcal{O}^{H}\left({ }^{3} P_{2}^{(8)}\right)\right\rangle \\
& =\frac{\pi^{3} \alpha_{s}^{2}}{M^{3}} \frac{80}{27} \delta\left(\hat{s}-M^{2}\right)\left\langle\mathcal{O}^{H}\left({ }^{3} P_{0}^{(8)}\right)\right\rangle .
\end{aligned}
$$


In deriving the last equation, we used the heavy quark spin symmetry [38],

$$
\left\langle\mathcal{O}^{H}\left({ }^{3} P_{J}^{(8)}\right)\right\rangle=(2 J+1)\left\langle\mathcal{O}^{H}\left({ }^{3} P_{0}^{(8)}\right)\right\rangle .
$$

From Eqs. (39) and (40), after we neglect the color singlet channels that have wrong spin and angular momentum quantum numbers, the gluonic contribution from the initial-state and final-state rescattering to the direct production of $H\left({ }^{3} S_{1}\right)$ are effectively the same except the sign of the $i \epsilon$ for the unpinched poles,

$$
\begin{aligned}
& H_{g g \rightarrow H}^{(I)}=\frac{8 \pi^{2} \alpha_{s}}{N_{c}^{2}-1}\left[\frac{1}{2 \pi} \frac{1}{x_{1}-x-i \epsilon} \frac{1}{x_{2}-x+i \epsilon}\right] C_{A} \hat{\sigma}_{g g}^{(0)}, \\
& H_{g g \rightarrow H}^{(F)}=\frac{8 \pi^{2} \alpha_{s}}{N_{c}^{2}-1}\left[\frac{1}{2 \pi} \frac{1}{x_{1}-x+i \epsilon} \frac{1}{x_{2}-x-i \epsilon}\right] C_{A} \hat{\sigma}_{g g}^{(0)},
\end{aligned}
$$

where the gluon-gluon fusion cross section is defined as

$$
\hat{\sigma}_{g g}^{(0)} \equiv \frac{\pi^{3} \alpha_{s}^{2}}{M^{3}} \frac{5}{12} \delta\left(\hat{s}-M^{2}\right)\left[\left\langle\mathcal{O}^{H}\left({ }^{1} S_{0}^{(8)}\right)\right\rangle+\frac{7}{m_{Q}^{2}}\left\langle\mathcal{O}^{H}\left({ }^{3} P_{0}^{(8)}\right)\right\rangle\right] .
$$

In deriving Eq. (48), the heavy quark spin symmetry was used to relate the matrix elements of different $P$-wave states to the matrix element of $\left\langle\mathcal{O}^{H}\left({ }^{3} P_{0}^{(8)}\right)\right\rangle$.

Substituting the partonic cross sections in Eqs. (43), (45), and (48) into Eq. (33), and integrating over gluon momentum fractions, $x_{1}$ and $x_{2}$, by taking the residue, we obtain the double scattering contribution to the $q_{T}^{2}$-moment of heavy quarkonium production in NRQCD model as,

$$
\begin{array}{r}
\int d q_{T}^{2} q_{T}^{2} \frac{d \sigma_{h A \rightarrow H}^{D}}{d q_{T}^{2}}=\left[\frac{8 \pi^{2} \alpha_{s}}{N_{c}^{2}-1}\right]\left(\sum_{q} \int d x^{\prime} \phi_{\bar{q} / h}\left(x^{\prime}\right) \int d x\left[T_{q / A}^{(I)}(x) C_{F} \hat{\sigma}_{q \bar{q}}^{(0)}+T_{q / A}^{(F)}(x)\left(C_{A} \hat{\sigma}_{q \bar{q}}^{(0)}+\hat{\sigma}_{q \bar{q}}^{(1)}\right)\right]\right. \\
\left.+\int d x^{\prime} \phi_{g / h}\left(x^{\prime}\right) \int d x\left[T_{g / A}^{(I)}(x) C_{A} \hat{\sigma}_{g g}^{(0)}+T_{g / A}^{(F)}(x) C_{A} \hat{\sigma}_{g g}^{(0)}\right]\right)
\end{array}
$$

which has a very similar expression as that in Eq. (23) derived in CEM in last subsection.

If we use the same model for the quark-gluon and gluon-gluon correlation functions as that used in CEM calculation in last subsection, we obtain the heavy quarkonium broadening in NRQCD model as

$$
\Delta\left\langle q_{T}^{2}\right\rangle_{\mathrm{HQ}}^{\mathrm{NRQCD}}=\left(\frac{8 \pi^{2} \alpha_{s}}{N_{c}^{2}-1} \lambda^{2} A^{1 / 3}\right) \frac{\left(C_{F}+C_{A}\right) \sigma_{q \bar{q}}^{(0)}+2 C_{A} \sigma_{g g}^{(0)}+\sigma_{q \bar{q}}^{(1)}}{\sigma_{q \bar{q}}^{(0)}+\sigma_{g g}^{(0)}}
$$

where the leading order cross sections calculated in NRQCD model are given by

$$
\begin{aligned}
\sigma_{q \bar{q}}^{(0,1)} & =\sum_{q} \int d x^{\prime} \phi_{\bar{q} / h}\left(x^{\prime}\right) \int d x \phi_{q / A}(x) \hat{\sigma}_{q \bar{q}}^{(0,1)}, \\
\sigma_{g g}^{(0)} & =\int d x^{\prime} \phi_{g / h}\left(x^{\prime}\right) \int d x \phi_{g / A}(x) \hat{\sigma}_{g g}^{(0)},
\end{aligned}
$$

with the partonic cross sections given in Eqs. (44), (46), and (49), respectively.

From the transverse momentum broadening in Eq. (28) calculated in CEM in last subsection and that in Eq. (51) calculated in NRQCD model, it is clear that the leading double scattering contribution to the broadening calculated in these two models have the same expression if one neglects the $P$-wave contribution in NRQCD approach. Since the $P$-wave contribution is smaller than the $S$-wave contribution, and the gluon-gluon fusion subprocess dominates the heavy quarkonium cross section, we expect that $\Delta\left\langle q_{T}^{2}\right\rangle_{\mathrm{HQ}}^{\mathrm{NRQCD}} \approx \Delta\left\langle q_{T}^{2}\right\rangle_{\mathrm{HQ}}^{\mathrm{CEM}} \approx\left(2 C_{A} / C_{F}\right) \Delta\left\langle q_{T}^{2}\right\rangle_{\mathrm{DY}}$.

In both CEM and NRQCD approach to the production of quarkonia, $H\left({ }^{3} S_{1}\right)$, we can also calculate the broadening effect on those quarkonia that were produced from the decay of either excited or high spin states of produced quarkonia, known as the feeddown mechanism of the quarkonium production. Since the $q_{T}^{2}$-moment in Eq. (1) is normalized by the cross section (the $0^{\text {th }}$-moment), and the rescattering takes place at the parton-level, we expect that the feed-down mechanism is not very sensitive to the quarkonium broadening while it is much more sensitive to the quarkonium production rate. We will come back to the role of the feeddown mechanism in quarkonium broadening in Sec. $\mathrm{V}$ when we present our numerical results. 


\section{Transverse momentum broadening in nucleus-nucleus collisions}

In this subsection, we extend our calculations of heavy quarkonium's transverse momentum broadening in hadronnucleus collisions to the broadening in nucleus-nucleus collisions. We discuss the similarities and differences between the hadron-nucleus and nucleus-nucleus collisions, and the role of transverse momentum broadening in probing the properties of the dense and hot QCD matter created in high energy nucleus-nucleus collisions.

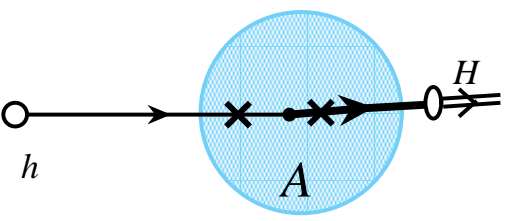

(a)

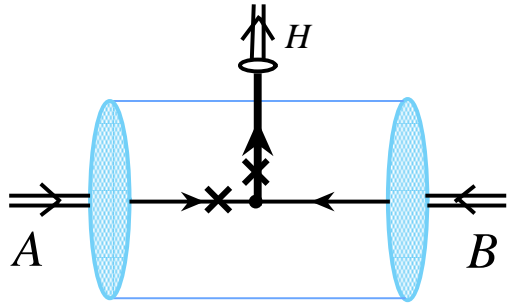

(b)

FIG. 6: Sketch of heavy quarkonium production in hadron-nucleus collisions as viewed in the target rest frame (a), and that in nucleus-nucleus collisions as viewed in the center-of-mass frame (b). The thin and thick lines indicate the incoming parton and the outgoing heavy quark pair, respectively. The cross indicates potential rescattering point with soft partons of the nuclear medium.

The broadening of heavy quarkonium's transverse momentum is a consequence of both initial-state and final-state rescattering in nuclear medium. In high energy hadron-nucleus collisions, both initial-state and final-state rescattering probe the same properties of a normal nuclear matter. If the scattering process is viewed in the rest frame of the nucleus, as sketched in Fig. 6(a), the incoming active parton and the produced heavy quark pair move very fast along the direction of the incoming hadron and interact with only partons of the nucleus near the same impact parameter. This picture supports our approximation that the correlation functions for final-state interaction are about the same as that for initial-state interaction. If the same process is viewed in the center of mass frame, we get the same conclusion that only nuclear partons near the same impact parameter can participate in the rescattering because the nuclear matter is moving very fast in this frame.

However, in high energy nucleus-nucleus collisions, the situation can be very different. If, other than the hard parton-parton scattering to produce the heavy quark pair and corresponding rescattering discussed above, other partons from two colliding nuclei did not interact in the collision, the transverse momentum broadening of heavy quarkonium production would be a simple superposition of the broadening in hadron-nucleus collision. In this picture, the leading contribution to the broadening in a collision between a nucleus $A$ and a nucleus $B$ would be given by the same expression in Eq. (31) with the $A^{1 / 3}$ replaced by $A^{1 / 3}+B^{1 / 3}$ or more precisely by $L_{A B} / r_{0}$, where $r_{0} \approx 0.8$ fm and $L_{A B}$ is an effective medium length in nucleus-nucleus collision [39]. The value of $r_{0}$ is obtained by letting $L_{p A} \approx r_{0} A^{1 / 3} \approx(3 / 4) R_{A}$ with the nuclear radius $R_{A}$.

But, as indicated by the early data from RHIC, soft partons from two nuclei do interact to form a dense and hot QCD quark-gluon medium in high energy nucleus-nucleus collisions. The final-state rescattering between the produced heavy quark pair of transverse momentum $q_{T}$ and the almost stationary or slowly expanding hot QCD medium in the center of mass frame of nucleus-nucleus collision, as sketched in Fig. 6(b), is very unlikely to broaden the pair's transverse momentum. Instead, the final-state interaction could suppress the production rate of the leading (or large momentum) colored and coherent heavy quark pair due to the energy loss [23], which is responsible for the observed jet quenching (or the suppression of leading hadrons or heavy quarks) at RHIC [1], and decrease the averaged transverse momentum $\left\langle q_{T}^{2}\right\rangle$. On the other hand, the initial-state interaction in nucleus-nucleus collisions is likely to broaden the transverse momentum of the active parton in the same way as that in hadron-nucleus collisions. Therefore, the measured $\left\langle q_{T}^{2}\right\rangle$ in nucleus-nucleus collision is a consequence of two competing effects: the initial-state interaction tries to broaden the transverse momentum while the final-state rescattering in a slowly expanding medium tends to reduce the pair's transverse momentum. The detailed analysis and calculation of the competing final-state effects on the quarkonium broadening in nucleus-nucleus collisions requires a careful modeling of the hot and dense medium and is beyond the scope of this paper.

Precise measurements of transverse momentum broadening of heavy quarkonium production in relativistic heavy ion collisions should provide very valuable information on the formation of the dense and hot quark-gluon medium and its properties. In nucleus-nucleus collisions, a deviation of the transverse momentum broadening from the simple superposition of that measured in hadron-nucleus collisions clearly indicates a change of nuclear matter properties from the interaction between soft partons of colliding nuclei. It should indicate the formation of a dense quark-gluon 
medium when the measured transverse momentum broadening is equal or less than the expected broadening from the initial-state interaction alone.

If the long-range interaction of soft partons from two colliding nuclei is quick and strong, and the dense quark-gluon medium is formed very early in relativistic heavy ion collisions, the initial-state interaction in nucleus-nucleus collisions could be different from a superposition of the initial-state effect in hadron-nucleus collisions due to the modification of nuclear matter. In order to independently test the initial-state effect from the final-state rescattering, we calculate the transverse momentum broadening of $Z$ (as well as $W$ ) bosons in relativistic heavy ion collisions at the LHC in next section.

\section{TRANSVERSE MOMENTUM BROADENING OF $Z$ (AND $W$ ) PRODUCTION AT THE LHC}

The lack of the final-state interaction of a $Z$ (or a $W$ ) boson when it is reconstructed from its leptonic decay channels makes its transverse momentum broadening in high energy nuclear collisions an ideal probe for the initialstate interaction, in particular, the density of nuclear medium in the early stage of relativistic heavy ion collisions [25]. If the long-range soft gluon interactions between two incoming heavy ions were so strong and a dense nuclear medium was formed before the short-distance creation of a $Z$ (or a $W$ ) boson, the transverse momentum broadening would be a clean probe of the density of the dense medium. Otherwise, the transverse momentum broadening of $Z$ (or $W$ ) bosons in nucleus-nucleus collisions would be a simple superposition of that in hadron-nucleus collision. Therefore, by measuring the broadening of $Z$ (or $W$ ) bosons in both hadron-nucleus and nucleus-nucleus collisions, we could learn valuable information on whether the dense quark-gluon medium could be formed at a very early stage in relativistic heavy ion collisions [24].

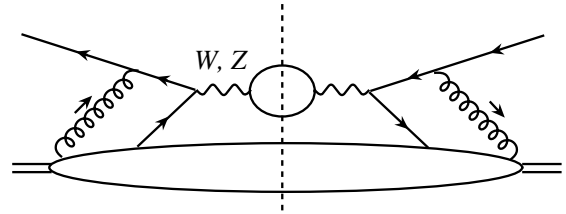

(a)

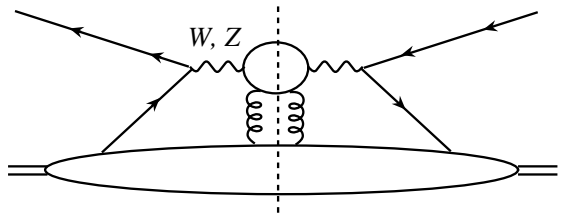

(b)

FIG. 7: Leading order double scattering diagrams for production of a $Z$ (or a $W$ ) boson in hadron-nucleus collisions via the initial-state interaction (a) and the possible final-state rescattering if the vector boson is reconstructed from its hadronic decay.

In Fig. 7] we sketch the leading double scattering diagrams for the $Z$ (or $W$ ) production in hadron-nucleus collisions. The diagram in Fig. 7(a), which is almost identical to that for the Drell-Yan transverse momentum broadening, represents the initial-state interaction, while that in Fig. 7)(b) represents the possible final-state rescattering if the vector boson could be reconstructed from its hadronic decay channels. For the $Z$ and $W$ bosons reconstructed from their leptonic decay channels, their transverse momentum broadening is mainly caused by the initial-state interaction, just like the broadening of the virtual photon in the Drell-Yan production. From the leading double scattering diagram in Fig. 7(a) and following the same derivation for the Drell-Yan broadening in Sec. II, we obtain the leading transverse momentum broadening of $Z$ (or $W$ ) bosons in hadron-nucleus collisions as,

$$
\begin{aligned}
\Delta\left\langle q_{T}^{2}\right\rangle_{h A}^{Z} & =C_{F} \frac{8 \pi^{2} \alpha_{s}\left(M_{Z}\right)}{N_{c}^{2}-1} \lambda_{Z}^{2} A^{1 / 3} \\
\Delta\left\langle q_{T}^{2}\right\rangle_{h A}^{W} & =C_{F} \frac{8 \pi^{2} \alpha_{s}\left(M_{W}\right)}{N_{c}^{2}-1} \lambda_{W}^{2} A^{1 / 3}
\end{aligned}
$$

where $\lambda_{Z}^{2}\left(\lambda_{W}^{2}\right)$ is the nonperturbative scale for the double scattering production of $Z$ (or $W$ ) bosons. It is defined in the same was as that in Eq.(13) and represents a ratio of nuclear four parton correlation function over a corresponding normal parton distribution. It is proportional to an averaged gluon field strength square in nuclear matter, $\left\langle F^{+\alpha} F_{\alpha}^{+}\right\rangle$ [40]. The $\lambda_{Z}$ should be proportional to the typical transverse momentum or effective virtuality of soft gluons participating in the double scattering. As we will discuss in next section, the value of the $\lambda_{Z}^{2}$ (or $\lambda_{W}^{2}$ ) could depend on the momentum exchange of the hard collision, $Q \sim M_{Z}$ (or $M_{W}$ ), as well as the collision energy.

If we assume that the dense quark-gluon medium in relativistic heavy ion collisions is not formed before the creation of the heavy vector boson $Z$ (or $W$ ), we could apply our formula in Eq. (53) to the broadening in nucleus-nucleus collisions as a simple superposition of the hadron-nucleus collision, and obtain the transverse momentum broadening 
in nucleus-nucleus collision as

$$
\Delta\left\langle q_{T}^{2}\right\rangle_{A B}^{V} \approx C_{F} \frac{8 \pi^{2} \alpha_{s}\left(M_{V}\right)}{N_{c}^{2}-1} \lambda_{V}^{2} \frac{L_{A B}}{r_{0}}
$$

where $V=Z, W$ for the $Z$ and $W$ production, respectively. If the long-range soft gluon interactions between two colliding heavy ions are so strong that the dense quark-gluon medium was formed before the short-distance creation of a $Z$ (or a $W$ ) boson, we expect the same formula in Eq. (54) to be valid for the leading contribution to the transverse momentum broadening, but, with a new $\lambda_{V}^{2}$ different from that of a normal nuclear matter. The value of the effective $\lambda_{V}^{2}$ and its dependence on the collision geometry and collision energy should provide valuable informations on the formation of the QCD medium and its properties.

\section{NUMERICAL RESULTS}

In this section, we provide a numerical comparison between our calculated heavy quarkonium broadening in nuclear collisions with existing data from Fermilab and RHIC experiments, and make predictions for the transverse momentum broadening at the LHC.

We have calculated in this paper the transverse momentum broadening of vector boson production in nuclear collisions in terms of the QCD factorization approach. We factorized the rescattering contribution to the broadening into two parts: (1) the non-perturbative, but, well-defined universal parton-parton correlation functions, which represent the probability to find the scattering centers in the nuclear medium, and (2) corresponding parton-level rescattering subprocess, which are infrared safe and perturbatively calculable. As a result of the factorization, the normalization for the transverse momentum broadening from partonic double scattering is uniquely fixed by the size of the non-perturbative quark-gluon and gluon-gluon correlation functions. If we use the model in Eqs. (13) and (27) to parameterize the correlation functions, the numerical results of our calculated transverse momentum broadening should be directly proportional to the value of the $\lambda^{2}$.

The value of the $\lambda^{2}$, or more precisely, the value of the parton-parton correlation functions should not depend on which vector boson was produced. This is because of the universality and the long-distance nature of the correlation functions. However, the value of the correlation functions or the $\lambda^{2}$ should depend on the momentum scale at which the hard part of the partonic scattering was evaluated.

As explicitly demonstrated in last three sections, all phase space integrations for the partonic rescattering can be deformed into perturbative region, and both initial- and final-state parton-level rescattering are evaluated at a hard scale $Q \sim 2 m_{Q}$ or $M_{Z}$ (or $M_{W}$ ) for heavy quarkonium or $Z$ (or $W$ ) production, respectively. From the model in Eq. (13) (or (27)), the non-perturbative parameter, $\lambda^{2}$, represents a ratio of nuclear four parton correlation function over a normal parton distribution. As demonstrated in Ref. [40] by approximately decomposing a nuclear state into a product of nucleon states, the ratio (so as the $\lambda^{2}$ ) can be expressed in terms of an averaged gluon field strength square, $\left\langle F^{+\alpha} F_{\alpha}^{+}\right\rangle$. In this picture, the $\lambda$ represents the virtuality or the typical transverse momentum of the partons participating in the partonic rescattering, and certainly depends on the momentum exchange of the hard collision, $Q$, as well as the collision energy, $\sqrt{s}$, which determines the available phase space for the collision. For hadron-nucleus collisions with a large momentum transfer, $Q$, we expect the $\lambda^{2}$ to be proportional to $\ln \left(Q^{2}\right)$ from the parton shower leading to the hard collision. If the collision energy $\sqrt{s}$ is very large and the momentum fraction of the active parton, $x$, is very small, we would expect the $\lambda^{2}$ to be proportional to the saturation scale $Q_{s}^{2} \propto 1 / x^{\delta}$ with $\delta \approx 0.3[41$, 42 ]. For the vector boson production, the typical momentum fraction of an active parton, $x \sim Q / \sqrt{s}$. Therefore, we expect $\lambda^{2}(Q) \propto \ln \left(Q^{2}\right)(\sqrt{s} / Q)^{\delta}$.

We use data on the Drell-Yan broadening in hadron-nucleus collisions, which were measured by Fermilab experiments

at the fixed target energy, $\sqrt{s}=38.8 \mathrm{GeV}$ [15], to extract the $\lambda_{\mathrm{DY}}^{2}$. From the value of $\lambda_{\mathrm{DY}}^{2}$, we estimate the value of $\lambda^{2}$ for producing a vector boson of invariant mass $Q$ at a collision energy $\sqrt{s}$ as follows,

$$
\lambda^{2}(Q) \approx \lambda_{\mathrm{DY}}^{2} \frac{\ln \left(Q^{2}\right)}{\ln \left(\left\langle Q_{\mathrm{DY}}^{2}\right\rangle\right)}\left(\frac{\sqrt{s} / Q}{38.8 /\left\langle Q_{\mathrm{DY}}\right\rangle}\right)^{\delta},
$$

with the $\sqrt{s}$ in unit of $\mathrm{GeV},\left\langle Q_{\mathrm{DY}}\right\rangle \sim 6 \mathrm{GeV}$, and $\delta \sim 0.3$.

Fermilab experiments: E772, E789, and E866 have measured the transverse momentum broadening of the DrellYan, as well as $\mathrm{J} / \psi, \psi^{\prime}$, and $\Upsilon$ production in hadron-nucleus collisions [15, 22]. In Fig. 8, we plot the data on both the Drell-Yan and heavy quarkonium broadening as a function of atomic weight of nuclear targets. The broadening for the data was defined as a difference between the $q_{T}^{2}$-moment in proton-nucleus and proton-deuteron collisions: $\Delta\left\langle q_{T}^{2}\right\rangle=\left\langle q_{T}^{2}\right\rangle_{p A}-\left\langle q_{T}^{2}\right\rangle_{p D}$. By fitting the data on the Drell-Yan broadening as a function of $A^{1 / 3}-2^{1 / 3}$, we obtain 


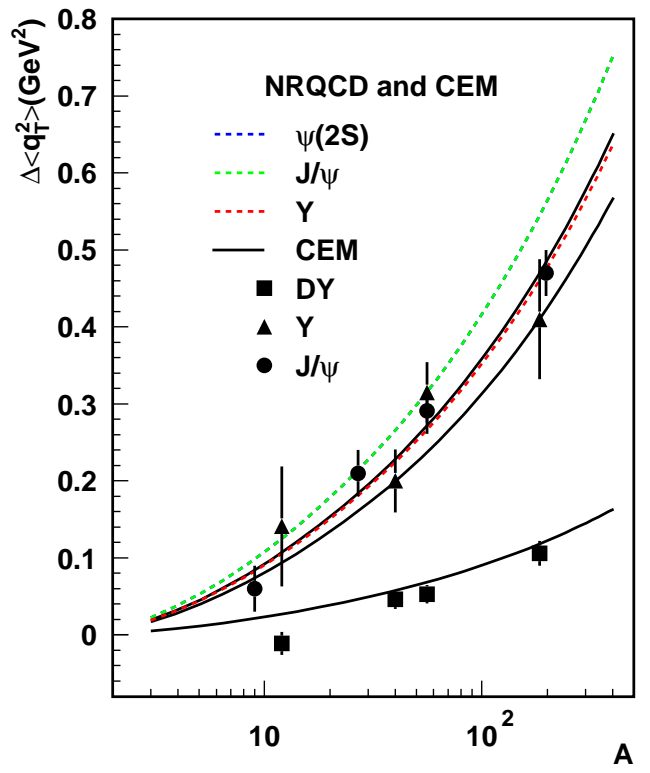

(a)

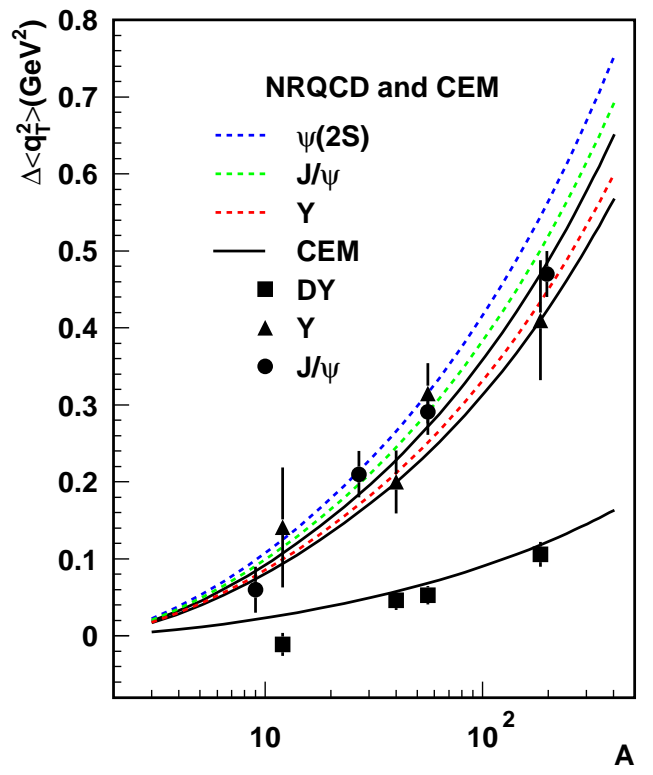

(b)

FIG. 8: Data on transverse momentum broadening of heavy quarkonium as well as Drell-Yan production in hadron-nucleus collisions. Also plotted are theoretical calculations using Eq. (28) (solid lines) and Eq. (51) (dashed lines), derived by using CEM and NRQCD model, respectively. Three solid lines (from the top to bottom) correspond to $J / \psi, \Upsilon$, and Drell-Yan, while three dashed lines represent $\psi^{\prime}, \mathrm{J} / \psi$, and $\Upsilon$ production from NRQCD model. The quarkonium broadening calculated in NRQCD model is evaluated with (a) and without (b) quarkonia from the feeddown mechanism.

$\lambda_{\mathrm{DY}}^{2} \approx 0.01 \mathrm{GeV}^{2}$, which gives the bottom solid line for the Drell-Yan broadening in Fig. 8 , and is consistent with the value extracted in Ref. [13].

In Fig. 8(a), we plot our theoretical calculations of transverse momentum broadening of direct heavy quarkonium production in hadron-nucleus collisions at the Fermilab fixed target energy, $\sqrt{s}=38.8 \mathrm{GeV}$. To obtain the numerical results of theoretical calculations, we use CTEQ6L for nucleon parton distribution functions [43] and EKS98 parametrization for nuclear parton distributions (nPDF) [44] to evaluate the leading order production cross sections in Eqs. (28) and (51). The non-perturbative parameter, $\lambda^{2}$, in Eqs. (28) and (51) could be slightly different for $\mathrm{J} / \psi$ and $\Upsilon$ production due to the difference in the scale of hard collision, $Q \sim 2 m_{Q}$. Using $\lambda_{\mathrm{DY}}^{2} \approx 0.01 \mathrm{GeV}^{2}$, $M_{\mathrm{J} / \psi}=3.1 \mathrm{GeV}, M_{\Upsilon}=9.5 \mathrm{GeV}$, we estimate from Eq. (55) that $\lambda_{\mathrm{J} / \psi}^{2} \approx 0.008 \mathrm{GeV}^{2}$ and $\lambda_{\Upsilon}^{2} \approx 0.011 \mathrm{GeV}^{2}$ at $\sqrt{s}=38.8 \mathrm{GeV}$. We use Eq. (51) to evaluate the broadening for $\psi^{\prime}, \mathrm{J} / \psi$, and $\Upsilon$ (the dashed lines) in NRQCD model. The NRQCD matrix elements are taken from Refs. [4, 45]. The small difference between the broadening of $\mathrm{J} / \psi$ and $\Upsilon$ is caused by the relative size between the quark and gluon contribution due to different sizes of matrix elements and the range of parton momentum fractions. For the direct production, $\mathrm{J} / \psi$ and $\psi^{\prime}$ have almost the identical broadening as shown in Fig. 8(a). Similarly, we use Eq. (28) to evaluate the broadening for J/ $\psi$ (top solid line) and $\Upsilon$ (middle solid line) in CEM with $m_{Q}=1.5 \mathrm{GeV}$ and $M_{Q}=1.85 \mathrm{GeV}$ for $\mathrm{J} / \psi$ and $m_{Q}=4.5 \mathrm{GeV}$ and $M_{Q}=5.3 \mathrm{GeV}$ for $\Upsilon$ production, respectively. The transition probability, $F_{Q \bar{Q} \rightarrow H}$, cancels between the numerator and the denominator in Eq. (28). The small difference between these two solid lines are again caused by the relative size of the quark and gluon contribution due to the slightly different ranges of parton momentum fractions for $\mathrm{J} / \psi$ and $\Upsilon$ production.

We also test the effect of transverse momentum broadening on heavy quarkonia produced by the feeddown mechanism. Using the partonic hard parts derived in Sec. IIIB we calculate in NRQCD model the transverse momentum broadening of quarkonia in high spin or excited states, which then decay into the observed spin-1 and $S$-wave quarkonia, $H\left({ }^{3} H_{1}\right)$. We plot the calculated broadening with this feeddown mechanism in Fig. 8 (b). Although the feeddown mechanism could provide as much as $40 \%$ of $\mathrm{J} / \psi$ production rate, its net effect on the transverse momentum broadening is very small because of the fact that the broadening defined in Eq. (1) is normalized by the inclusive cross section.

For a large nucleus with the atomic weight $A \geq 10^{2}$, some of the produced heavy quark pairs could transmute to a color singlet pre-quarkonium state (or even a physical quarkonium) before exiting the nuclear matter. Comparing to a colored heavy quark pair, these color singlet states should have a weaker interaction with the nuclear matter and get less broadening in transverse momentum. Therefore, we expect the theoretical curves in Fig. 8 to be slightly less steep than what were shown when $A \geq 10^{2}$. 
From Fig. 8, we conclude that perturbative QCD calculations of the quarkonium broadening based on both CEM and NRQCD model give a good description of existing experimental data in hadron-nucleus collisions. The major difference between the heavy quarkonium and the Drell-Yan broadening is naturally explained by the role of final-state interactions. Although the production mechanism in CEM and NRQCD model is different, these two models of heavy quarkonium production predict almost the same functional form for the transverse momentum broadening, as shown in Eq. (28) and Eq. (51), respectively. Since the $P$-wave contribution is relatively small, as shown in Fig. 8 , these two models predict almost the same transverse momentum broadening. In addition, both models predict that $\mathrm{J} / \psi$ and $\Upsilon$ have effectively the same broadening in hadron-nucleus collisions other than a small difference caused by the available phase space (i.e., the available range of parton momentum fractions).

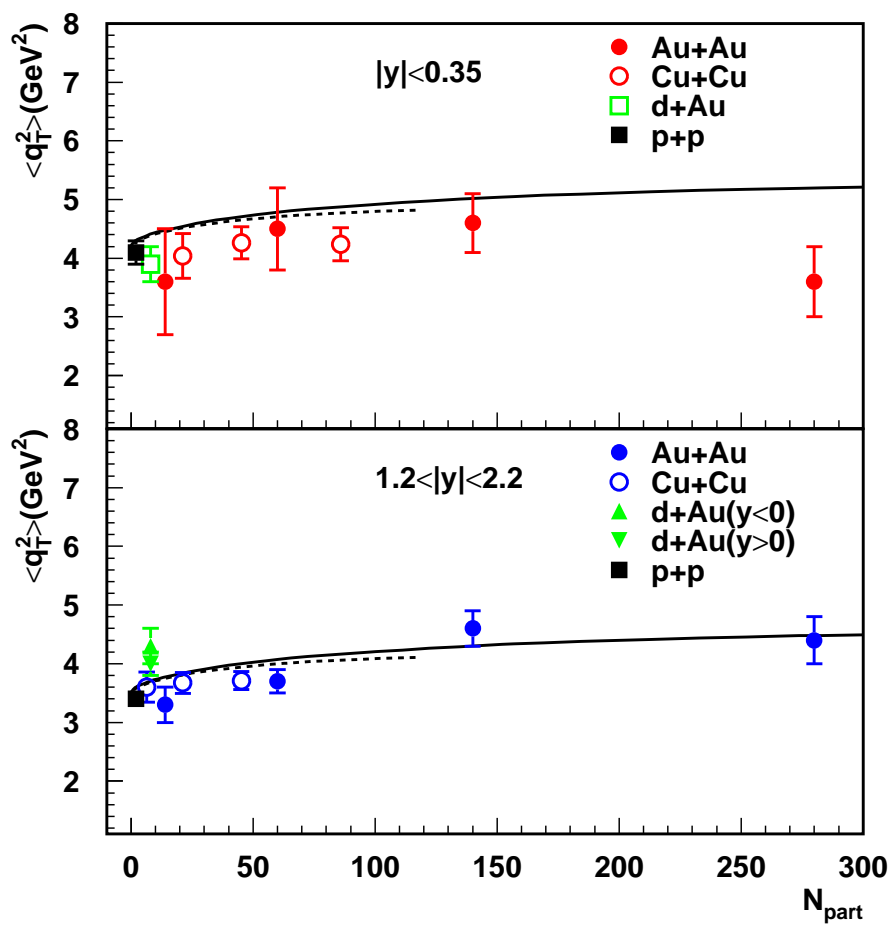

FIG. 9: RHIC data on averaged transverse momentum square of $\mathrm{J} / \psi$ production as a function of the number of participants [46]. The top panel is for the $\mathrm{J} / \psi$ 's produced in the central rapidity region while the bottom panel is for those produced in more forward or backward region. Also plotted are theoretical calculations using Eq. (56). Solid lines for Au-Au collisions and dashed lines for $\mathrm{Cu}-\mathrm{Cu}$ collisions, respectively.

In Fig. 9] we plot PHENIX data on averaged transverse momentum square of $\mathrm{J} / \psi$ production at RHIC energies as a function of the number of participants, $\mathrm{N}_{\text {part }}$ [46]. The top panel is for the $\mathrm{J} / \psi$ produced in the central rapidity region with $|y|<0.35$, and the bottom is for those produced in the forward and backward region with $1.2<|y|<2.2$. We also plot our theoretical calculations of the transverse momentum square by using

$$
\left.\left\langle q_{T}^{2}\right\rangle_{A B} \approx\left\langle q_{T}^{2}\right\rangle\right|_{p p-e x p}+\Delta\left\langle q_{T}^{2}\right\rangle_{A B}
$$

where $\left.\left\langle q_{T}^{2}\right\rangle\right|_{p p-e x p}$ is fixed by the data point from proton-proton collision in Fig. 9 and $\Delta\left\langle q_{T}^{2}\right\rangle_{A B}$ is given by our calculation. We evaluate $\Delta\left\langle q_{T}^{2}\right\rangle_{A B}$ in nucleus-nucleus collisions with an effective medium length $L_{A B}$ as

$$
\Delta\left\langle q_{T}^{2}\right\rangle_{A B \rightarrow \mathrm{J} / \psi}^{\mathrm{CEM}}=\left(\frac{8 \pi^{2} \alpha_{s}}{N_{c}^{2}-1} \lambda_{\mathrm{J} / \psi}(\mathrm{RHIC})^{2} \frac{L_{A B}}{r_{0}}\right) \frac{\left(C_{F}+C_{A}\right) \sigma_{q \bar{q}}+2 C_{A} \sigma_{g g}}{\sigma_{q \bar{q}}+\sigma_{g g}}
$$

in CEM. Eq. (57) is obtained from Eq. (28) by replacing the $A^{1 / 3}$ by $L_{A B} / r_{0}$. We can calculate the broadening in NRQCD model by applying the same replacement to Eq. (51). At the RHIC energy, we obtain $\lambda_{\mathrm{J} / \psi}(\mathrm{RHIC})^{2} \approx$ $0.013 \mathrm{GeV}^{2}$ from Eq. (55). We calculate the $L_{A B}$ in Glauber model and convert it to $\mathrm{N}_{\text {part }}$ [39].

In nucleus-nucleus collisions, soft gluons from the colliding ions can interact even if the collision is not a head-on or a central collision. Such interaction in a non-central collision may not be strong enough to stop all soft partons to form an almost stationary or slowly expanding hot medium of quarks and gluons. It certainly can slow down some of the colliding soft partons to change the characteristics of the nuclear matter, which could alter the final-state 
interactions. As a result, the final-state interaction between the produced heavy quark pairs and the modified nuclear matter in nucleus-nucleus collisions, as sketched in Fig. 6(b), generates less transverse momentum broadening if it does not reduce the transverse momentum due to energy loss. In Fig. 9, we plot our calculations of $\mathrm{J} / \psi$ transverse momentum broadening by using Eqs. (56) and (57), and keeping only the contribution from initial-state rescattering. The solid lines are for the $\mathrm{Au}-\mathrm{Au}$ collision, while the dashed lines are for the $\mathrm{Cu}-\mathrm{Cu}$ collision. Our calculations are consistent with the data in both rapidity regions.

In the central $\mathrm{Au}-\mathrm{Au}$ collision, a hot and dense medium is produced. As discussed above, the averaged transverse momentum could be reduced, instead of the broadening, due to the energy loss of the produced heavy quark pairs when they interact with the hot and slowly expanding medium. A more detailed study of the momentum shift of the heavy quark pairs in such a hot medium could provide a more accurate description of the data in central region, and help the extraction of medium properties.

Transverse momentum broadening of $Z$ (or $W$ ) bosons in high energy nuclear collisions could provide a clean measurement of initial-state interactions, and help isolating final-state rescattering effect in heavy quarkonium production. However, because of the heavy mass of $Z$ (or $W$ ) boson, only the LHC has a chance to measure the broadening reliably 24].

We use Eq. (55) to estimate the $\lambda^{2}$ for the heavy vector boson production at $\sqrt{s}=5.5 \mathrm{TeV}$, the averaged nucleonnucleon collision energy in relativistic heavy ion collisions at the LHC. We obtain $\lambda^{2}(\mathrm{LHC}) \approx 0.035,0.05$ and $0.05 \mathrm{GeV}^{2}$ for the production of $\mathrm{J} / \psi, \Upsilon$, and the heavy vector boson $Z$ (or $W$ ), respectively. Although the $\Upsilon$ mass is much smaller than that of a $Z$ boson, the $\Upsilon$ and $Z$ have the same $\lambda^{2}$ (LHC) for the transverse momentum broadening due to the larger available phase space for $\Upsilon$ production [47]. In Fig. [10, we present our predictions for the transverse momentum broadening of vector boson production at the LHC. Using the estimated $\lambda_{Z / W}^{2}(\mathrm{LHC}) \approx 0.05 \mathrm{GeV}^{2}$ and Eq. (53), we evaluate the transverse momentum broadening of $Z$ (and $W$ ) bosons reconstructed from their leptonic decays, and plot the predictions for hadron-nucleus collisions as a function of atomic weight of the nucleus, $A$, in Fig. 10(a). We also plot the expected transverse momentum broadening of $\mathrm{J} / \psi$ and $\Upsilon$ production in hadron-nucleus collisions in Fig. 10(a). The curves for heavy quarkonium broadening are evaluated by using $\lambda_{\mathrm{J} / \psi}^{2}(\mathrm{LHC}) \approx 0.035 \mathrm{GeV}^{2}$ and $\lambda_{\Upsilon}^{2}(\mathrm{LHC}) \approx 0.05 \mathrm{GeV}^{2}$, and Eq. (28) from CEM without contributions from the feeddown mechanism. Eq. (51) derived from NRQCD model gives the similar results. The heavy quarkonium broadening in Fig. 10(a) is much larger than that of $Z$ (or $W$ ) bosons because of the additional final-state effect, and the difference in color factor and the strength of the strong coupling constant, $\alpha_{s}(Q)$.

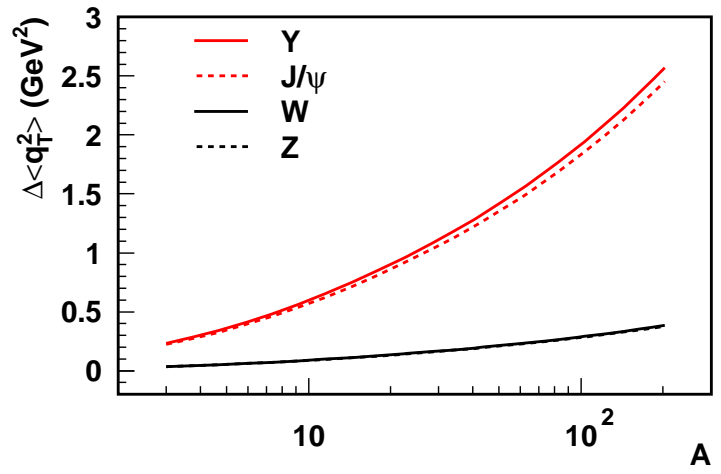

(a)

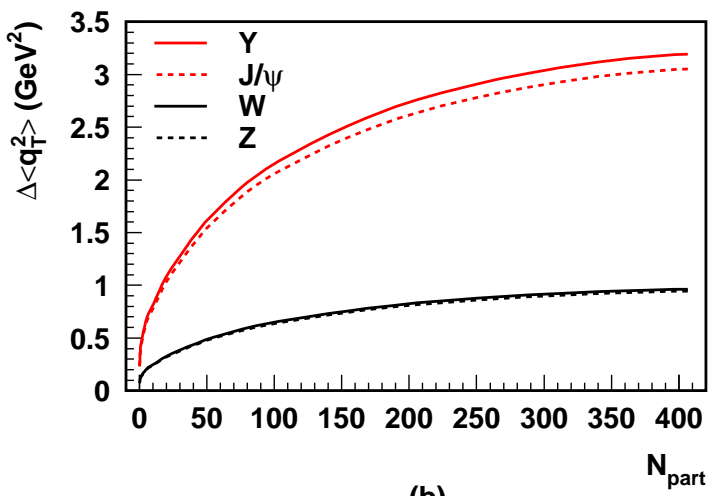

(b)

FIG. 10: Transverse momentum broadening of $Z$ and $W$ (lower set of curves) in hadron-nucleus collisions (a) and nucleusnucleus collisions (b) at $\sqrt{s}=5.5 \mathrm{TeV}$ as a function of atomic weight of nuclear beam and the number of participants, $\mathrm{N}_{\text {part }}$, respectively. Also plotted are predictions (upper set of curves) for the transverse momentum broadening of heavy quarkonium production in hadron-nucleus collision at the LHC energy (a); and the initial-state only broadening in nucleus-nucleus collision at the same energy (b).

In Fig. 10(b), we plot the expected transverse momentum broadening of vector boson production in $\mathrm{Pb}-\mathrm{Pb}$ collision at $\sqrt{s}=5.5 \mathrm{TeV}$ at the LHC as a function of the number of participants, $\mathrm{N}_{\text {part }}$ [39]. We calculate the $L_{A B}$ in Glauber model with inelastic nucleon-nucleon cross section $\sigma_{N N}^{i n}=70 \mathrm{mb}$ at the LHC energy and convert it to $\mathrm{N}_{\text {part }}$ in $\mathrm{Pb}-\mathrm{Pb}$ collisions [39]. For the $Z$ (and $W$ ) broadening, we use $\lambda_{Z / W}(\mathrm{LHC})^{2} \approx 0.05 \mathrm{GeV}^{2}$, the same value used for the broadening in hadron-nucleus collisions in Fig. 10(a). Since the transverse momentum broadening is directly proportional to the $\lambda^{2}$, which is proportional to the gluon strength in the medium, a deviation from the predicted curves in Fig. 10(b) signals the formation of the hot quark-gluon medium before the creation of the heavy $Z$ (or $W$ ) bosons. 
For $\mathrm{J} / \psi$ and $\Upsilon$ production in Fig. 10 (b), we use $\lambda_{\mathrm{J} / \psi}^{2}(\mathrm{LHC}) \approx 0.035 \mathrm{GeV}^{2}$ and $\lambda_{\Upsilon}^{2}(\mathrm{LHC}) \approx 0.05 \mathrm{GeV}^{2}$, and Eq. (28) from CEM to evaluate the quarkonium broadening. Eq. (51) from NRQCD model gives similar predictions. The plotted curves for $\mathrm{J} / \psi$ and $\Upsilon$ production in Fig. 10(b) include only initial-state interaction. As discussed in Sec. IIIC the final-state rescattering in nucleus-nucleus collisions is more likely to reduce the transverse momentum broadening due to the energy loss, if a slowly expanding medium was produced. Therefore, we expect the curves in Fig. 10(b) to represent the maximum broadening of $\mathrm{J} / \psi$ and $\Upsilon$ that will be seen in relativistic heavy ion collisions at the LHC [24].

If we could reconstruct $Z$ and $W$ bosons from their hadronic decay (e.g., into two jets), which might be impossible to do in the LHC environment, the hadronic jets from $Z$ and $W$ decay will have to interact with the nuclear medium. The final-state multiple scattering could generate momentum imbalance between these two jets and effectively introduce an apparent mass shift for the $Z$ and $W$ boson [9]. Such mass shift would provide additional information on the properties of the hot quark-gluon medium.

\section{SUMMARY}

In this paper we evaluated transverse momentum broadening of vector boson production in hadron-nucleus and nucleus-nucleus collisions in terms of parton-level multiple scattering. We argued that the broadening, $\Delta\left\langle q_{T}^{2}\right\rangle_{A B} \equiv$ $\left\langle q_{T}^{2}\right\rangle_{A B}-\left\langle q_{T}^{2}\right\rangle_{h h}$, defined as a difference between the averaged transverse momentum square measured in nuclear collisions and that in hadron-hadron collisions, can be systematically calculated in perturbative QCD in terms of the collinear factorization approach [7]. We factorize the contribution to transverse momentum broadening into the calculable short-distance partonic rescattering multiplied by universal parton-parton correlation functions, which specify the probabilities to find coherent scattering centers in a nuclear medium. We explicitly show that the partonic rescattering diagrams are evaluated at a perturbative hard scale $Q \sim 2 m_{Q}$. We verify the universality of the non-perturbative parton-parton correlation functions by fitting the data on both the Drell-Yan broadening and the broadening of $\mathrm{J} / \psi$ and $\Upsilon$ production to clearly demonstrate the predictive power of the QCD factorization approach.

For the Drell-Yan virtual photon and $Z$ (or $W$ ) boson production, we evaluated transverse momentum broadening from purely initial-state multiple scattering. We discussed the scale dependence of the non-perturbative parameter, $\lambda^{2}$, and extrapolate it from its value at the fixed-target energy to the collider energies. We calculated the broadening of $Z$ (and $W$ ) bosons in both hadron-nucleus and nucleus-nucleus collisions at the LHC. We evaluated the $Z$ (and $W$ ) boson broadening in nucleus-nucleus collisions as a superpostion of its broadening in hadron-nucleus collisions, and argued that a deviation from our calculation is a clear signal indicating that strong interactions between soft partons of colliding heavy ions took place before the short-distance creation of $Z$ bosons. That is, the transverse momentum broadening of $Z$ (or $W$ ) bosons could be a clean and excellent probe of the early stage dynamics of relativistic heavy ion collisions.

For $\mathrm{J} / \psi$ and $\Upsilon$ production, we demonstated that the final-state interaction between the produced heavy quark pair and the nuclear medium is very important in understanding the existing data. We calculated the broadening in both CEM and NRQCD model, and clearly showed that the two models generate a small difference in the broadening and the broadening has a weak dependence on the feeddown mechanism. That is, our results are insensitive to the details of the hadronization mechanism and perturbatively reliable. We found that the leading contribution to heavy quarkonium broadening in hadron-nucleus collisions is about $2 C_{A} / C_{F}=4.5$ times the corresponding Drell-Yan broadening, which gives a good description of the existing Fermilab data.

We argued that the role of the final-state interaction to the transverse momentum broadening in nucleus-nucleus collisions could be very different from that in hadron-nucleus collisions. In hadron-nucleus collisions, both initial-state and final-state interactions involve only soft partons of the nucleus near the same impact parameter, and therefore, provide similar contributions to the transverse momentum broadening as long as the heavy quark pair stays in a colored state. On the other hand, soft partons from two colliding nuclei could strongly interact to produce a slowly expanding quark-gluon medium. This new medium could interact with the produced heavy quark pair if the pair is in a color octet state, and the interaction could be very weak or vanish if the pair is in a singlet state. The final-state interaction with this new medium in nucleus-nucleus collision, as sketched in Fig. [6]could reduce (instead, to broaden) the pair's transverse momentum.

We predicted the transverse momentum broadening of vector boson $(\mathrm{J} / \psi, \Upsilon$, and $W / Z)$ production in hadronnucleus collisions and a maximum broadening in nucleus-nucleus collisions at the LHC in Fig. 10, A more detailed calculation of final-state interaction between the produced heavy quark pair and the dense and slowly expanding quark-gluon medium produced in nucleus-nucleus collisions could provide a better conncetion between the measured average of the transverse momentum square and the properties of the dense quark-gluon medium in relativistic heavy ion collisions [48]. Having a precise transverse momentum broadening could also shed some lights on nonperturbative dynamics that determines the transverse momentum distribution of vector boson production in low $q_{T}$ region [10]. 
Finally, we emphasize that our QCD factorization approach for calculating the heavy quarkonium broadening can be applied for studying the nuclear dependence of quarkonium cross sections in high energy nuclear collisions [5], as well as the nuclear dependence of the quarkonium's rapidity and transverse momentum distributions if the transverse momentum $q_{T}$ is large enough [7, 8]. The advantage of the QCD factorization formalism is that we may readily quantify corrections in powers of $\alpha_{s}$.

\section{Acknowledgments}

We thank George Sterman for useful discussions on heavy quarkonium production. We thank M.J. Leitch and J.C. Peng for helpful correspondence on E772 and E866 data. J. Q. thanks the Theory Group of High Energy Physics Division at Argonne National Laboratory for its hospitality and support when a part of this work was completed. This work was supported in part by the U.S. Department of Energy under Grants No. DE-FG02-87ER40371 and Contract DE-AC02-06CH11357, and in part by the Argonne University of Chicago Joint Theory Institute (JTI) Grant 03921-07-137.

[1] I. Arsene et al. [BRAHMS Collaboration], Nucl. Phys. A 757, 1 (2005) arXiv:nucl-ex/0410020; B. B. Back et al., Nucl. Phys. A 757, 28 (2005) arXiv:nucl-ex/0410022]; J. Adams et al. [STAR Collaboration], Nucl. Phys. A 757, 102 (2005) arXiv:nucl-ex/0501009; K. Adcox et al. [PHENIX Collaboration], Nucl. Phys. A 757, 184 (2005) arXiv:nucl-ex/0410003.

[2] G. Boyd, J. Engels, F. Karsch, E. Laermann, C. Legeland, M. Lutgemeier and B. Petersson, Nucl. Phys. B 469, 419 (1996) arXiv:hep-lat/9602007]; F. Karsch, Nucl. Phys. A 698, 199 (2002) arXiv:hep-ph/0103314, and references therein; M. Cheng et al., Phys. Rev. D 74, 054507 (2006) arXiv:hep-lat/0608013.

[3] T. Matsui and H. Satz, Phys. Lett. B178, 416 (1986).

[4] For a review, see N. Brambilla et al. [Quarkonium Working Group], arXiv:hep-ph/0412158, and references therein.

[5] J. W. Qiu, J. P. Vary and X. f. Zhang, Phys. Rev. Lett. 88, 232301 (2002) arXiv:hep-ph/9809442]; Nucl. Phys. A 698, 571 (2002) arXiv:nucl-th/0106040.

[6] G. C. Nayak, J. W. Qiu and G. Sterman, Phys. Rev. Lett. 99, 212001 (2007) arXiv:0707.2973 [hep-ph]]; Phys. Rev. D (in press) arXiv:0711.3476 [hep-ph]].

[7] For a review, see J. W. Qiu and G. Sterman, Int. J. Mod. Phys. E 12, 149 (2003); J. W. Qiu, Eur. Phys. J. C 43, 239 (2005) arXiv:hep-ph/0507268.

[8] M. Luo, J. W. Qiu and G. Sterman, Phys. Lett. B 279 (1992) 377; Phys. Rev. D 50, 1951 (1994).

[9] M. Luo, J. W. Qiu and G. Sterman, Phys. Rev. D 49, 4493 (1994).

[10] X. f. Guo, J. W. Qiu and X. f. Zhang, Phys. Rev. Lett. 84, 5049 (2000) arXiv:hep-ph/9911476; Phys. Rev. D 62, 054008 (2000) arXiv:hep-ph/9912361.

[11] J. C. Collins and J. W. Qiu, Phys. Rev. D 75, 114014 (2007) arXiv:0705.2141 [hep-ph]].

[12] G. T. Bodwin, S. J. Brodsky and G. P. Lepage, Phys. Rev. D 39, 3287 (1989).

[13] X. f. Guo, Phys. Rev. D 58, 114033 (1998).

[14] R. J. Fries, Phys. Rev. D 68, 074013 (2003) arXiv:hep-ph/0209275.

[15] P. L. McGaughey, J. M. Moss and J. C. Peng, Annu. Rev. Nucl. Part. Sci. 49, 217 (1999); J. C. Peng, arXiv:hep-ph/9912371

[16] M. J. Leitch, Eur. Phys. J. C 43, (157-160) 2005.

[17] M. B. Johnson, B. Z. Kopeliovich, M. J. Leitch, P. L. McGaughey, J. M. Moss, I. K. Potashnikova and I. Schmidt, Phys. Rev. C 75, 035206 (2007) arXiv:hep-ph/0606126.

[18] S. J. Brodsky and A. H. Mueller, Phys. Lett. B 206, 685 (1988).

[19] G. T. Bodwin, E. Braaten and G. P. Lepage, Phys. Rev. D 51, 1125 (1995) [Erratum-ibid. D 55, 5853 (1997)] arXiv:hep-ph/9407339.

[20] M.B. Einhorn and S.D. Ellis, Phys. Rev. D12, 2007 (1975); H. Fritzsch, Phys. Lett. B67. 217 (1977); M. Glück, J.F. Owens and E. Reya, Phys. Rev. D17, 2324 (1978); J. Babcock, D. Sivers and S. Wolfram, Phys. Rev. D18, 162 (1978).

[21] C.-H. Chang, Nucl. Phys. B172, 425 (1980); E.L. Berger and D. Jones, Phys. Rev. D23, 1521 (1981); R. Baier and R. Rückl, Phys. Lett. B102, 364 (1981).

[22] E772 Collaboration, D.M. Alde et al., Phys. Rev. Lett. 66, 2285 (1991); E772 Collaboration, D.M. Alde et al., Phys. Rev. Lett. 64, 2479 (1990); E789 Collaboration, M.J. Leitch et al., Phys. Rev. D 52, 4251 (1995); M.A. Vasiliev et al., Phys. Rev. Lett. 83, 2304 (1999).

[23] Y. L. Dokshitzer and D. E. Kharzeev, Phys. Lett. B 519, 199 (2001) arXiv:hep-ph/0106202 ; M. Djordjevic and M. Gyulassy, Nucl. Phys. A 733, 265 (2004) arXiv:nucl-th/0310076.

[24] Z. B. Kang and J. W. Qiu, arXiv:0707.0276 [hep-ph].

[25] A. Accardi et al., arXiv:hep-ph/0308248, and references therein.

[26] J. C. Collins, D. E. Soper and G. Sterman, Adv. Ser. Direct. High Energy Phys. 5, 1 (1988) arXiv:hep-ph/0409313.

[27] E. L. Berger, J. W. Qiu and X. f. Zhang, Phys. Rev. D 65, 034006 (2002) arXiv:hep-ph/0107309.

[28] J. C. Collins, D. E. Soper and G. Sterman, Nucl. Phys. B 250, 199 (1985). 
[29] J. W. Qiu and X. f. Zhang, Phys. Rev. Lett. 86, 2724 (2001) arXiv:hep-ph/0012058; Phys. Rev. D 63, 114011 (2001) arXiv:hep-ph/0012348.

[30] N. Armesto, L. Cunqueiro, C. A. Salgado and W. C. Xiang, arXiv:0710.3073 [hep-ph].

[31] J. W. Qiu and G. Sterman, Nucl. Phys. B 353, 105 (1991); Nucl. Phys. B 353, 137 (1991).

[32] R. Gavai, D. Kharzeev, H. Satz, G. A. Schuler, K. Sridhar and R. Vogt, Int. J. Mod. Phys. A 10, 3043 (1995) arXiv:hep-ph/9502270.

[33] J. F. Amundson, O. J. P. Eboli, E. M. Gregores and F. Halzen, Phys. Lett. B 390, 323 (1997) arXiv:hep-ph/9605295.

[34] C. J. Benesh, J. W. Qiu and J. P. Vary, Phys. Rev. C 50, 1015 (1994) arXiv:hep-ph/9403265, and references therein.

[35] J. C. Collins, D. E. Soper and G. Sterman, Nucl. Phys. B 263, 37 (1986).

[36] G. C. Nayak, J. W. Qiu and G. Sterman, Phys. Lett. B 613, 45 (2005) arXiv:hep-ph/0501235; Phys. Rev. D 72, 114012 (2005) arXiv:hep-ph/0509021]; Phys. Rev. D 74, 074007 (2006) arXiv:hep-ph/0608066.

[37] E. Braaten, S. Fleming and T. C. Yuan, Ann. Rev. Nucl. Part. Sci. 46, 197 (1996) arXiv:hep-ph/9602374;

M. Kramer, Prog. Part. Nucl. Phys. 47, 141 (2001) arXiv:hep-ph/0106120; J. P. Lansberg, Int. J. Mod. Phys. A 21, 3857 (2006) arXiv:hep-ph/0602091.

[38] P. Cho and A. K. Leibovich, Phys. Rev. D 53, 150 (1996); P. Cho and A. K. Leibovich, Phys. Rev. D 53, 6203 (1996); M. Beneke and I. Z. Rothstein, Phys. Rev. D 54, 2005 (1996); P. Ko, J. Lee and H. S. Song, Phys. Rev. D 54, 4312 (1996).

[39] M. L. Miller, K. Reygers, S. J. Sanders and P. Steinberg, Ann. Rev. Nucl. Part. Sci. 57, 205 (2007) arXiv:nucl-ex/0701025.

[40] J. W. Qiu and I. Vitev, Phys. Rev. Lett. 93, 262301 (2004) arXiv:hep-ph/0309094.

[41] K. Golec-Biernat and M. Wusthoff, Phys. Rev. D 59, 014017 (1999).

[42] For a review, see E. Iancu and R. Venugopalan, arXiv:hep-ph/0303204, and references therein.

[43] J. Pumplin et al, JHEP 07, 012 (2002), hep-ph/0201195.

[44] K.J. Eskola, V.J. Kolhinen and C.A. Salgado, Eur. Phys. J. C 9, 61 (1999), hep-ph/9807297

[45] E. Braaten, S. Fleming and A. K. Leibovich, Phys. Rev. D 63, 094006 (2001) arXiv:hep-ph/0008091].

[46] A. Adare et al. [PHENIX Collaboration], Phys. Rev. Lett. 98, 232002 (2007) arXiv:hep-ex/0611020]; arXiv:0711.3917 [nucl-ex]; Phys. Rev. Lett. 98, 232301 (2007) arXiv:nucl-ex/0611020]; arXiv:0801.0220 [nucl-ex].

[47] E. L. Berger, J. W. Qiu and Y. l. Wang, Phys. Rev. D 71, 034007 (2005) arXiv:hep-ph/0404158.

[48] Z. B. Kang and J. W. Qiu, in preparation. 